\title{
On Tanaka's Prolongation Procedure for Filtered Structures of Constant Type
}

Igor ZELENKO

Department of Mathematics, Texas A\&M University, College Station, TX 77843-3368, USA

E-mail: zelenko@math.tamu.edu

Received June 02, 2009, in final form September 29, 2009; Published online October 06, 2009

doi:10.3842/SIGMA.2009.094

\begin{abstract}
We present Tanaka's prolongation procedure for filtered structures on manifolds discovered in [Tanaka N., J. Math. Kyoto. Univ. 10 (1970), 1-82] in a spirit of SingerSternberg's description of the prolongation of usual $G$-structures [Singer I.M., Sternberg S., J. Analyse Math. 15 (1965), 1-114; Sternberg S., Prentice-Hall, Inc., Englewood Cliffs, N.J., 1964]. This approach gives a transparent point of view on the Tanaka constructions avoiding many technicalities of the original Tanaka paper.
\end{abstract}

Key words: $G$-structures; filtered structures; generalized Spencer operator; prolongations

2000 Mathematics Subject Classification: 58A30; 58A17

\section{Introduction}

This note is based on series of lectures given by the author in the Working Geometry Seminar at the Department of Mathematics at Texas A\&M University in Spring 2009. The topic is the prolongation procedure for filtered structures on manifolds discovered by Noboru Tanaka in the paper [10] published in 1970. The Tanaka prolongation procedure is an ingenious refinement of Cartan's method of equivalence. It provides an effective algorithm for the construction of canonical frames for filtered structures, and for the calculation of the sharp upper bound of the dimension of their algebras of infinitesimal symmetries. This note is by no means a complete survey of the Tanaka theory. For such a survey we refer the reader to [5]. Our goal here is to describe geometric aspects of Tanaka's prolongation procedure using the language similar to one used by Singer and Sternberg in [7] and [9] for description of the prolongation of the usual $G$-structures. We found that it gives a quite natural and transparent point of view on Tanaka's constructions, avoiding many formal definitions and technicalities of the original Tanaka paper. We believe this point of view will be useful to anyone who is interested in studying both the main ideas and the details of this fundamental Tanaka construction. We hope that the material of Sections 3 and 4 will be of interest to experts as well. Our language also allows to generalize the Tanaka procedure in several directions, including filtered structures with non-constant and non-fundamental symbols. These generalizations, with applications to the local geometry of distributions, will be given in a separate paper.

\subsection{Statement of the problem}

Let $D$ be a rank $l$ distribution on a manifold $M$; that is, a rank $l$ subbundle of the tangent bundle $T M$. Two vector distributions $D_{1}$ and $D_{2}$ are called equivalent if there exists a diffeomorphism $F: M \rightarrow M$ such that $F_{*} D_{1}(x)=D_{2}(F(x))$ for any $x \in M$. Two germs

\footnotetext{
*This paper is a contribution to the Special Issue "Élie Cartan and Differential Geometry". The full collection is available at http://www.emis.de/journals/SIGMA/Cartan.html
} 
of vector distributions $D_{1}$ and $D_{2}$ at the point $x_{0} \in M$ are called equivalent, if there exist neighborhoods $U$ and $\tilde{U}$ of $x_{0}$ and a diffeomorphism $F: U \rightarrow \tilde{U}$ such that

$$
F_{*} D_{1}=D_{2}, \quad F\left(x_{0}\right)=x_{0} .
$$

The general question is: When are two germs of distributions equivalent?

\subsection{Weak derived flags and symbols of distributions}

Taking Lie brackets of vector fields tangent to a distribution $D$ (i.e. sections of $D$ ) one can define a filtration $D^{-1} \subset D^{-2} \subset \cdots$ of the tangent bundle, called a weak derived flag or a small flag (of $D$ ). More precisely, set $D=D^{-1}$ and define recursively $D^{-j}=D^{-j+1}+\left[D, D^{-j+1}\right]$, $j>1$. Let $X_{1}, \ldots X_{l}$ be $l$ vector fields constituting a local basis of a distribution $D$, i.e. $D=\operatorname{span}\left\{X_{1}, \ldots, X_{l}\right\}$ in some open set in $M$. Then $D^{-j}(x)$ is the linear span of all iterated Lie brackets of these vector fields, of length not greater than $j$, evaluated at a point $x$. A distribution $D$ is called bracket-generating (or completely nonholonomic) if for any $x$ there exists $\mu(x) \in \mathbb{N}$ such that $D^{-\mu(x)}(x)=T_{x} M$. The number $\mu(x)$ is called the degree of nonholonomy of $D$ at a point $x$. A distribution $D$ is called regular if for all $j<0$, the dimensions of subspaces $D^{j}(x)$ are independent of the point $x$. From now on we assume that $D$ is regular bracket-generating distribution with degree of nonholonomy $\mu$. Let $\mathfrak{g}^{-1}(x) \stackrel{\text { def }}{=} D^{-1}(x)$ and $\mathfrak{g}^{j}(x) \stackrel{\text { def }}{=} D^{j}(x) / D^{j+1}(x)$ for $j<-1$. Consider the graded space

$$
\mathfrak{m}(x)=\bigoplus_{j=-\mu}^{-1} \mathfrak{g}^{j}(x)
$$

corresponding to the filtration

$$
D(x)=D^{-1}(x) \subset D^{-2}(x) \subset \cdots \subset D^{-\mu+1}(x) \subset D^{-\mu}(x)=T_{x} M .
$$

This space is endowed naturally with the structure of a graded nilpotent Lie algebra, generated by $\mathfrak{g}^{-1}(x)$. Indeed, let $\mathfrak{p}_{j}: D^{j}(x) \mapsto \mathfrak{g}^{j}(x)$ be the canonical projection to a factor space. Take $Y_{1} \in \mathfrak{g}^{i}(x)$ and $Y_{2} \in \mathfrak{g}^{j}(x)$. To define the Lie bracket $\left[Y_{1}, Y_{2}\right]$ take a local section $\widetilde{Y}_{1}$ of the distribution $D^{i}$ and a local section $\widetilde{Y}_{2}$ of the distribution $D^{j}$ such that $\mathfrak{p}_{i}\left(\widetilde{Y}_{1}(x)\right)=Y_{1}$ and $\mathfrak{p}_{j}\left(\widetilde{Y}_{2}(x)\right)=Y_{2}$. It is clear that $\left[Y_{1}, Y_{2}\right] \in \mathfrak{g}^{i+j}(x)$. Put

$$
\left[Y_{1}, Y_{2}\right] \stackrel{\text { def }}{=} \mathfrak{p}_{i+j}\left(\left[\widetilde{Y}_{1}, \widetilde{Y}_{2}\right](x)\right) .
$$

It is easy to see that the right-hand side of (1.1) does not depend on the choice of sections $\widetilde{Y}_{1}$ and $\widetilde{Y}_{2}$. Besides, $\mathfrak{g}^{-1}(x)$ generates the whole algebra $\mathfrak{m}(x)$. A graded Lie algebra satisfying the last property is called fundamental. The graded nilpotent Lie algebra $\mathfrak{m}(x)$ is called the symbol of the distribution $D$ at the point $x$.

Fix a fundamental graded nilpotent Lie algebra $\mathfrak{m}=\bigoplus_{i=-\mu}^{-1} \mathfrak{g}^{i}$. A distribution $D$ is said to be of constant symbol $\mathfrak{m}$ or of constant type $\mathfrak{m}$ if for any $x$ the symbol $\mathfrak{m}(x)$ is isomorphic to $\mathfrak{m}$ as a nilpotent graded Lie algebra. In general this assumption is quite restrictive. For example, in the case of rank two distributions on manifolds with $\operatorname{dim} M \geq 9$, symbol algebras depend on continuous parameters, which implies that generic rank 2 distributions in these dimensions do not have a constant symbol. For rank 3 distributions with $\operatorname{dim} D^{-2}=6$ the same holds in the case $\operatorname{dim} M=7$ as was shown in [3]. Following Tanaka, and for simplicity of presentation, we consider here distributions of constant type $\mathfrak{m}$ only. One can construct the flat distribution $D_{\mathfrak{m}}$ of constant type $\mathfrak{m}$. For this let $M(\mathfrak{m})$ be the simply connected Lie group with the Lie algebra $\mathfrak{m}$ and let $e$ be its identity. Then $D_{\mathfrak{m}}$ is the left invariant distribution on $M(\mathfrak{m})$ such that $D_{\mathfrak{m}}(e)=\mathfrak{g}^{-1}$. 


\subsection{The bundle $P^{0}(\mathfrak{m})$ and its reductions}

To a distribution of type $\mathfrak{m}$ one can assign a principal bundle in the following way. Let $G^{0}(\mathfrak{m})$ be the group of automorphisms of the graded Lie algebra $\mathfrak{m}$; that is, the group of all automorphisms $A$ of the linear space $\mathfrak{m}$ preserving both the Lie brackets $(A([v, w])=[A(v), A(w)]$ for any $v, w \in \mathfrak{m})$ and the grading $\left(A\left(\mathfrak{g}^{i}\right)=\mathfrak{g}^{i}\right.$ for any $\left.i<0\right)$. Let $P^{0}(\mathfrak{m})$ be the set of all pairs $(x, \varphi)$, where $x \in M$ and $\varphi: \mathfrak{m} \rightarrow \mathfrak{m}(x)$ is an isomorphism of the graded Lie algebras $\mathfrak{m}$ and $\mathfrak{m}(x)$. Then $P^{0}(\mathfrak{m})$ is a principal $G^{0}(\mathfrak{m})$-bundle over $M$. The right action $R_{A}$ of an automorphism $A \in G^{0}(\mathfrak{m})$ is as follows: $R_{A}$ sends $(x, \varphi) \in P^{0}(\mathfrak{m})$ to $(x, \varphi \circ A)$, or shortly $(x, \varphi) \cdot R_{A}=(x, \varphi \circ A)$. Note that since $\mathfrak{g}^{-1}$ generates $\mathfrak{m}$, the group $G^{0}(\mathfrak{m})$ can be identified with a subgroup of $\mathrm{GL}\left(\mathfrak{g}^{-1}\right)$. By the same reason a point $(x, \varphi) \in P^{0}(\mathfrak{m})$ of a fiber of $P^{0}(\mathfrak{m})$ is uniquely defined by $\left.\varphi\right|_{\mathfrak{g}^{-1}}$. So one can identify $P^{0}(\mathfrak{m})$ with the set of pairs $(x, \psi)$, where $x \in M$ and $\psi: \mathfrak{g}^{-1} \rightarrow D(x)$ can be extended to an automorphism of the graded Lie algebras $\mathfrak{m}$ and $\mathfrak{m}(x)$. Speaking informally, $P^{0}(\mathfrak{m})$ can be seen as a $G^{0}(\mathfrak{m})$-reduction of the bundle of all frames of the distribution $D$. Besides, the Lie algebra $\mathfrak{g}^{0}(\mathfrak{m})$ is the algebra of all derivations $a$ of $\mathfrak{m}$, preserving the grading (i.e. $a \mathfrak{g}^{i} \subset \mathfrak{g}^{i}$ for all $i<0$ ).

Additional structures on distributions can be encoded by reductions of the bundle $P^{0}(\mathfrak{m})$. More precisely, let $G^{0}$ be a Lie subgroup of $G^{0}(\mathfrak{m})$ and let $P^{0}$ be a principal $G^{0}$-bundle, which is a reduction of the bundle $P^{0}(\mathfrak{m})$. Since $\mathfrak{g}^{0}$ is a subalgebra of the algebra of derivations of $\mathfrak{m}$ preserving the grading, the subspace $\mathfrak{m} \oplus \mathfrak{g}^{0}$ is endowed with the natural structure of a graded Lie algebra. For this we only need to define brackets $[f, v]$ for $f \in \mathfrak{g}^{0}$ and $v \in \mathfrak{m}$, because $\mathfrak{m}$ and $\mathfrak{g}^{0}$ are already Lie algebras. Set $[f, v] \stackrel{\text { def }}{=} f(v)$. The bundle $P^{0}$ is called a structure of constant type $\left(\mathfrak{m}, \mathfrak{g}^{0}\right)$. Let, as before, $D_{\mathfrak{m}}$ be the left invariant distribution on $M(\mathfrak{m})$ such that $D_{\mathfrak{m}}(e)=\mathfrak{g}^{-1}$. Denote by $L_{x}$ the left translation on $M(\mathfrak{m})$ by an element $x$. Finally, let $P^{0}\left(\mathfrak{m}, \mathfrak{g}^{0}\right)$ be the set of all pairs $(x, \varphi)$, where $x \in M(\mathfrak{m})$ and $\varphi: \mathfrak{m} \rightarrow \mathfrak{m}(x)$ is an isomorphism of the graded Lie algebras $\mathfrak{m}$ and $\mathfrak{m}(x)$ such that $\left(L_{x^{-1}}\right)_{*} \varphi \in G^{0}$. The bundle $P^{0}\left(\mathfrak{m}, \mathfrak{g}^{0}\right)$ is called the flat structure of constant type $\left(\mathfrak{m}, \mathfrak{g}^{0}\right)$. Let us give some examples.

Example 1. $G$-structures. Assume that $D=T M$. So $\mathfrak{m}=\mathfrak{g}^{-1}$ is abelian, $G^{0}(\mathfrak{m})=$ $\mathrm{GL}(\mathfrak{m})$, and $P^{0}(\mathfrak{m})$ coincides with the bundle $\mathcal{F}(M)$ of all frames on $M$. In this case $P^{0}$ is nothing but a usual $G^{0}$-structure.

Example 2. Contact distributions. Let $D$ be the contact distribution in $\mathbb{R}^{2 n+1}$. Its symbol $\mathfrak{m}_{\text {cont }, n}$ is isomorphic to the Heisenberg algebra $\eta_{2 n+1}$ with grading $\mathfrak{g}^{-1} \oplus \mathfrak{g}^{-2}$, where $\mathfrak{g}^{-2}$ is the center of $\eta_{2 n+1}$. Obviously, a skew-symmetric form $\Omega$ is well defined on $\mathfrak{g}^{-1}$, up to a multiplication by a nonzero constant. The group $G^{0}\left(\mathfrak{m}_{\mathrm{cont}, n}\right)$ of automorphisms of $\mathfrak{m}_{\text {cont }, n}$ is isomorphic to the group $\operatorname{CSP}\left(\mathfrak{g}^{-1}\right)$ of conformal symplectic transformations of $\mathfrak{g}^{-1}$, i.e. transformations preserving the form $\Omega$, up to a multiplication by a nonzero constant.

Example 3. Maximally nonholonomic rank 2 distributions in $\mathbb{R}^{5}$. Let $D$ be a rank 2 distribution in $\mathbb{R}^{5}$ with degree of nonholonomy equal to 3 at every point. Such distributions were treated by É. Cartan in his famous work [1]. In this case $\operatorname{dim} D^{-2} \equiv 3$ and $\operatorname{dim} D^{-3} \equiv 5$. The symbol at any point is isomorphic to the Lie algebra $\mathfrak{m}_{(2,5)}$ generated by $X_{1}, X_{2}, X_{3}, X_{4}$, and $X_{5}$ with the following nonzero products: $\left[X_{1}, X_{2}\right]=X_{3},\left[X_{1}, X_{3}\right]=X_{4}$, and $\left[X_{2}, X_{3}\right]=X_{5}$. The grading is given as follows:

$$
\mathfrak{g}^{-1}=\left\langle X_{1}, X_{2}\right\rangle, \quad \mathfrak{g}^{-2}=\left\langle X_{3}\right\rangle, \quad \mathfrak{g}^{-3}=\left\langle X_{4}, X_{5}\right\rangle,
$$

where $\left\langle Y_{1}, \ldots, Y_{k}\right\rangle$ denotes the linear span of vectors $Y_{1}, \ldots, Y_{k}$. Since $\mathfrak{m}_{(2,5)}$ is a free nilpotent Lie algebra with two generators $X_{1}$ and $X_{2}$, its group of automorphism is equivalent to $\mathrm{GL}\left(\mathfrak{g}^{-1}\right)$.

Example 4. Sub-Riemannian structures of constant type (see also [6]). Assume that each space $D(x)$ is endowed with an Euclidean structure $Q_{x}$ depending smoothly on $x$. In this situation the pair $(D, Q)$ defines a sub-Riemannian structure on a manifold $M$. Recall that $\mathfrak{g}^{-1}(x)=D(x)$. This motivates the following definition: A pair $(\mathfrak{m}, \mathfrak{Q})$, where $\mathfrak{m}=\bigoplus_{j=-\mu}^{-1} \mathfrak{g}^{j}$ 
is a fundamental graded Lie algebra and $\mathfrak{Q}$ is an Euclidean structure on $\mathfrak{g}^{-1}$, is called a subRiemannian symbol. Two sub-Riemannian symbols $(\mathfrak{m}, \mathfrak{Q})$ and $(\tilde{\mathfrak{m}}, \widetilde{\mathfrak{Q}})$ are isomorphic if there exists a map $\varphi: \mathfrak{m} \rightarrow \tilde{\mathfrak{m}}$, which is an isomorphism of the graded Lie algebras $\mathfrak{m}$ and $\tilde{\mathfrak{m}}$, preserving the Euclidean structures $\mathfrak{Q}$ and $\tilde{\mathfrak{Q}}$ (i.e. such that $\widetilde{Q}\left(\varphi\left(v_{1}\right), \varphi\left(v_{2}\right)\right)=Q\left(v_{1}, v_{2}\right)$ for any $v_{1}$ and $v_{2}$ in $\left.\mathfrak{g}^{-1}\right)$. Fix a sub-Riemannian symbol $(\mathfrak{m}, \mathfrak{Q})$. A sub-Riemannian structure $(D, Q)$ is said to be of constant type $(\mathfrak{m}, \mathfrak{Q})$, if for every $x$ the sub-Riemannian symbol $\left(\mathfrak{m}(x), Q_{x}\right)$ is isomorphic to $(\mathfrak{m}, \mathfrak{Q})$.

It may happen that a sub-Riemannian structure does not have a constant symbol even if the distribution does. Such a situation occurs already in the case of the contact distribution on $\mathbb{R}^{2 n+1}$ for $n>1$ (see Example 2 above). As was mentioned above, in this case a skew-symmetric form $\Omega$ is well defined on $\mathfrak{g}^{-1}$, up to a multiplication by a nonzero constant. If in addition a Euclidean structure $Q$ is given on $\mathfrak{g}^{-1}$, then a skew-symmetric endomorphism $J$ of $\mathfrak{g}^{-1}$ is well defined, up to a multiplication by a nonzero constant, by $\Omega\left(v_{1}, v_{2}\right)=Q\left(J v_{1}, v_{2}\right)$. Take $0<\beta_{1} \leq \cdots \leq \beta_{n}$ so that $\left\{ \pm \beta_{1} i, \ldots, \pm \beta_{n} i\right\}$ is the set of the eigenvalues of $J$. Then a subRiemannian symbol with $\mathfrak{m}=\mathfrak{m}_{\text {cont }, n}$ is determined uniquely (up to an isomorphism) by a point $\left[\beta_{1}: \beta_{2}: \ldots: \beta_{n}\right]$ of the projective space $\mathbb{R P}^{n-1}$.

Let $(D, Q)$ be a sub-Riemannian structure of constant type $(\mathfrak{m}, \mathfrak{Q})$ and $G^{0}(\mathfrak{m}, \mathfrak{Q}) \subset G^{0}(\mathfrak{m})$ be the group of automorphisms of a sub-Riemannian symbol $(\mathfrak{m}, \mathfrak{Q})$. Let $P^{0}(\mathfrak{m}, \mathfrak{Q})$ be the set of all pairs $(x, \varphi)$, where $x \in M$ and $\varphi: \mathfrak{m} \rightarrow \mathfrak{m}(x)$ is an isomorhism of sub-Riemannian symbols $(\mathfrak{m}, \mathfrak{Q})$ and $\left(\mathfrak{m}(x), Q_{x}\right)$. Obviously, the bundle $P^{0}(\mathfrak{m}, \mathfrak{Q})$ is a reduction of $P^{0}(\mathfrak{m})$ with the structure group $G^{0}(\mathfrak{m}, \mathfrak{Q})$.

Example 5. Second order ordinary differential equations up to point transformations. Assume that $D$ is a contact distribution on a 3-dimensional manifold endowed with two distinguished transversal line sub-distributions $L_{1}$ and $L_{2}$. Such structures appear in the study of second order ordinary differential equations $y^{\prime \prime}=F\left(t, y, y^{\prime}\right)$ modulo point transformations. Indeed, let $J^{i}(\mathbb{R}, \mathbb{R})$ be the space of $i$-jets of mappings from $\mathbb{R}$ to $\mathbb{R}$. As the distribution $D$ we take the standard contact distribution on $J^{1}(\mathbb{R}, \mathbb{R})$. In the standard coordinates $(t, y, p)$ on $J^{1}(\mathbb{R}, \mathbb{R})$ this distribution is given by the Pfaffian equation $d y-p d t=0$. The natural lifts to $J^{1}$ of solutions of the differential equation form the 1-foliation tangent to $D$. The tangent lines to this foliation define the sub-distribution $L_{1}$. In the coordinates $(t, y, p)$ the sub-distribution $L_{1}$ is generated by the vector field $\frac{\partial}{\partial t}+p \frac{\partial}{\partial y}+F(t, y, p) \frac{\partial}{\partial p}$. Finally, consider the natural bundle $J^{1}(\mathbb{R}, \mathbb{R}) \rightarrow J^{0}(\mathbb{R}, \mathbb{R})$ and let $L_{2}$ be the distribution of the tangent lines to the fibers. The sub-distribution $L_{2}$ is generated by the vector field $\frac{\partial}{\partial p}$. The triple $\left(D, L_{1}, L_{2}\right)$ is called the pseudo-product structure associated with the second order ordinary differential equation. Two second order differential equations are equivalent with respect to the group of point transformations if and only if there is a diffeomorphism of $J^{1}(\mathbb{R}, \mathbb{R})$ sending the pseudo-product structure associated with one of them to the pseudo-product structure associated with the other one. This equivalence problem was treated by É. Cartan in [2] and earlier by A. Tresse in [12] and [13]. The symbol of the distribution is $\mathfrak{m}_{\text {cont, } 1} \sim \eta_{3}$ (see Example 2 above) and the plane $\mathfrak{g}^{-1}$ is endowed with two distinguished transversal lines. This additional structure is encoded by the subgroup $G^{0}$ of the group $G^{0}\left(\mathfrak{m}_{\text {cont,1 }}\right)$ preserving each of these lines.

Another important class of geometric structures that can be encoded in this way are $C R$ structures (see $\S 10$ of [10] for more details).

\subsection{Algebraic and geometric Tanaka prolongations}

In [10] Tanaka solves the equivalence problem for structures of constant type $\left(\mathfrak{m}, \mathfrak{g}^{0}\right)$. Two of Tanaka's main constructions are the algebraic prolongation of the algebra $\mathfrak{m}+\mathfrak{g}^{0}$, and the geometric prolongation of structures of type $\left(\mathfrak{m}, \mathfrak{g}^{0}\right)$, imitated by the algebraic prolongation. 
First he defines a graded Lie algebra, which is in essence the maximal (nondegenerated) graded Lie algebra, containing the graded Lie algebra $\bigoplus_{i \leq 0} \mathfrak{g}^{i}$ as its non-positive part. More precisely, Tanaka constructs a graded Lie algebra $\mathfrak{g}\left(\mathfrak{m}, \mathfrak{g}^{0}\right)=\bigoplus_{i \in \mathbb{Z}} \mathfrak{g}^{i}\left(\mathfrak{m}, \mathfrak{g}^{0}\right)$, satisfying the following three conditions:

1. $\mathfrak{g}^{i}\left(\mathfrak{m}, \mathfrak{g}^{0}\right)=\mathfrak{g}^{i}$ for all $i \leq 0$;

2. if $X \in \mathfrak{g}^{i}\left(\mathfrak{m}, \mathfrak{g}^{0}\right)$ with $i>0$ satisfies $\left[X, \mathfrak{g}^{-1}\right]=0$, then $X=0$;

3. $\mathfrak{g}\left(\mathfrak{m}, \mathfrak{g}^{0}\right)$ is the maximal graded Lie algebra, satisfying Properties 1 and 2.

This graded Lie algebra $\mathfrak{g}\left(\mathfrak{m}, \mathfrak{g}^{0}\right)$ is called the algebraic universal prolongation of the graded Lie algebra $\mathfrak{m} \oplus \mathfrak{g}^{0}$. An explicit realization of the algebra $\mathfrak{g}\left(\mathfrak{m}, \mathfrak{g}^{0}\right)$ will be described later in Section 4. It turns out $([10, \S 6],[14, \S 2])$ that the Lie algebra of infinitesimal symmetries of the flat structure of type $\left(\mathfrak{m}, \mathfrak{g}^{0}\right)$ can be described in terms of $\mathfrak{g}\left(\mathfrak{m}, \mathfrak{g}^{0}\right)$. If $\operatorname{dim} \mathfrak{g}\left(\mathfrak{m}, \mathfrak{g}^{0}\right)$ is finite (which is equivalent to the existence of $l>0$ such that $\mathfrak{g}^{l}\left(\mathfrak{m}, \mathfrak{g}^{0}\right)=0$ ), then the algebra of infinitesimal symmetries is isomorphic to $\mathfrak{g}\left(\mathfrak{m}, \mathfrak{g}^{0}\right)$. The analogous formulation in the case when $\mathfrak{g}\left(\mathfrak{m}, \mathfrak{g}^{0}\right)$ is infinite dimensional may be found in $[10, \S 6]$.

Furthermore for a structure $P^{0}$ of type $\left(\mathfrak{m}, \mathfrak{g}^{0}\right)$, Tanaka constructs a sequence of bundles $\left\{P^{i}\right\}_{i \in \mathbb{N}}$, where $P^{i}$ is a principal bundle over $P^{i-1}$ with an abelian structure group of dimension equal to $\operatorname{dim} \mathfrak{g}^{i}\left(\mathfrak{m}, \mathfrak{g}^{0}\right)$. In general $P^{i}$ is not a frame bundle. This is the case only for $\mathfrak{m}=\mathfrak{g}^{-1}$; that is, for $G$-structures. But if $\operatorname{dim} \mathfrak{g}\left(\mathfrak{m}, \mathfrak{g}^{0}\right)$ is finite or, equivalently, if there exists $l \geq 0$ such that $\mathfrak{g}^{l+1}\left(\mathfrak{m}, \mathfrak{g}^{0}\right)=0$, then the bundle $P^{l+\mu}$ is an $e$-structure over $P^{l+\mu-1}$, i.e. $P^{l+\mu-1}$ is endowed with a canonical frame (a structure of absolute parallelism). Note that all $P^{i}$ with $i \geq l$ are identified one with each other by the canonical projections (which are diffeomorphisms in that case). Hence, $P^{l}$ is endowed with a canonical frame. Once a canonical frame is constructed the equivalence problem for structures of type $\left(\mathfrak{m}, \mathfrak{g}^{0}\right)$ is in essence solved. Moreover, $\operatorname{dim} \mathfrak{g}\left(\mathfrak{m}, \mathfrak{g}^{0}\right)$ gives the sharp upper bound for the dimension of the algebra of infinitesimal symmetries of such structures.

By Tanaka's geometric prolongation we mean his construction of the sequence of bundles $\left\{P^{i}\right\}_{i \in \mathbb{N}}$. In this note we mainly concentrate on a description of this geometric prolongation using a language different from Tanaka's original one. In Section 2 we review the prolongation of usual $G$-structures in the language of Singer and Sternberg. We do this in order to prepare the reader for the next section, where the first Tanaka geometric prolongation is given in a completely analogous way. We believe that after reading Section 3 the reader will already have an idea how to proceed with the higher order Tanaka prolongations so that technicalities of Section 4 can be easily overcome.

\section{Review of prolongation of $G$-structures}

Before treating the general case we review the prolongation procedure for structures with $\mathfrak{m}=\mathfrak{g}^{-1}$, i.e. for usual $G$-structures. We follow [7] and [9]. Let $\Pi_{0}: P^{0} \rightarrow M$ be the canonical projection and $V(\lambda) \subset T_{\lambda} P^{0}$ the tangent space at $\lambda$ to the fiber of $P^{0}$ over the point $\Pi_{0}(\lambda)$. The subspace $V(\lambda)$ is also called the vertical subspace of $T_{\lambda} P^{0}$. Actually,

$$
V(\lambda)=\operatorname{ker}\left(\Pi_{0}\right)_{*}(\lambda)
$$

Recall that the space $V(\lambda)$ can be identified with the Lie algebra $\mathfrak{g}^{0}$ of $G^{0}$. The identification $I_{\lambda}: \mathfrak{g}^{0} \rightarrow V(\lambda)$ sends $X \in \mathfrak{g}^{0}$ to $\left.\frac{d}{d t}\left(\lambda \cdot R_{e^{t X}}\right)\right|_{t=0}$, where $e^{t X}$ is the one-parametric subgroup 
generated by $X$. Recall also that an Ehresmann connection on the bundle $P^{0}$ is a distribution $H$ on $P^{0}$ such that

$$
T_{\lambda} P^{0}=V(\lambda) \oplus H(\lambda) \quad \forall \lambda \in P^{0} .
$$

A subspace $H(\lambda)$, satisfying (2.2), is a horizontal subspace of $T_{\lambda} P^{0}$.

Once an Ehresmann connection $H$ and a basis in the space $\mathfrak{g}^{-1} \oplus \mathfrak{g}^{0}$ are fixed, the bundle $P^{0}$ is endowed with a frame in a canonical way. Indeed, let $\lambda=(x, \varphi) \in P^{0}$. Then $\varphi \in \operatorname{Hom}\left(\mathfrak{g}^{-1}, T_{x} M\right)$. By (2.1) and (2.2) the restriction $\left.\left(\Pi_{0}\right)_{*}\right|_{H(\lambda)}$ of the map $\left(\Pi_{0}\right)_{*}$ to the subspace $H(\lambda)$ is an isomorphism between $H(\lambda)$ and $T_{\Pi_{0}(\lambda)} M$. Define the map $\varphi^{H(\lambda)}: \mathfrak{g}^{-1} \oplus \mathfrak{g}^{0} \rightarrow$ $T_{\lambda} P^{0}$ as follows:

$$
\begin{aligned}
& \left.\varphi^{H(\lambda)}\right|_{\mathfrak{g}^{-1}}=\left(\left.\left(\Pi_{0}\right)_{*}\right|_{H(\lambda)}\right)^{-1} \circ \varphi, \\
& \left.\varphi^{H(\lambda)}\right|_{\mathfrak{g}^{0}}=I_{\lambda} .
\end{aligned}
$$

If one fixes a basis in $\mathfrak{g}^{-1} \oplus \mathfrak{g}^{0}$, then the images of this basis under the maps $\varphi^{H(\lambda)}$ define the frame (the structure of the absolute parallelism) on $P^{0}$.

The question is whether an Ehresmann connection can be chosen canonically. To answer this question, first one introduces a special $\mathfrak{g}^{-1}$-valued 1-form $\omega$ on $P^{0}$ as follows: $\omega(Y)=\varphi^{-1} \circ$ $\left(\Pi_{0}\right)_{*}(Y)$ for any $\lambda=(x, \varphi) \in P^{0}$ and $Y \in T_{\lambda} P^{0}$. This 1-form is called the soldering (tautological, fundamental) form of the $G^{0}$-structure $P^{0}$. Further, fixing again a point $\lambda=(x, \varphi) \in P^{0}$, one defines a structure function (a torsion) $C_{H} \in \operatorname{Hom}\left(\mathfrak{g}^{-1} \wedge \mathfrak{g}^{-1}, \mathfrak{g}^{-1}\right)$ of a horizontal subspace $H$ of $T_{\lambda} P^{0}$, as follows:

$$
\forall v_{1}, v_{2} \in \mathfrak{g}^{-1} \quad C_{H}\left(v_{1}, v_{2}\right)=-d \omega\left(\varphi^{H}\left(v_{1}\right), \varphi^{H}\left(v_{2}\right)\right),
$$

where $\varphi^{H}$ is defined by (2.3). Equivalently,

$$
C_{H}\left(v_{1}, v_{2}\right)=\omega\left(\left[Y_{1}, Y_{2}\right](\lambda)\right)
$$

for any vector fields $Y_{1}$ and $Y_{2}$ such that $\omega\left(Y_{i}\right) \equiv v_{i}$ and $\varphi^{H}\left(v_{i}\right)=Y_{i}(\lambda), i=1,2$. Speaking informally, the structure function $C_{H}$ encodes all information about horizontal parts at $\lambda$ of Lie brackets of vector fields which are horizontal at $\lambda$ w.r.t. the splitting (2.2) (with $H(\lambda)$ replaced by $H)$.

We now take another horizontal subspace $\widetilde{H}$ of $T_{\lambda} P^{0}$ and compare the structure functions $C_{H}$ and $C_{\widetilde{H}}$. By construction, for any vector $v \in \mathfrak{g}^{-1}$ the vector $\varphi^{\widetilde{H}}(v)-\varphi^{H}(v)$ belongs to $V(\lambda)$ $\left(\sim \mathfrak{g}^{0}\right)$. Let

$$
f_{H \widetilde{H}}(v) \stackrel{\text { def }}{=} I_{\lambda}^{-1}\left(\varphi^{\widetilde{H}}(v)-\varphi^{H}(v)\right) .
$$

Then $f_{H \widetilde{H}} \in \operatorname{Hom}\left(\mathfrak{g}^{-1}, \mathfrak{g}^{0}\right)$. In the opposite direction, it is clear that for any $f \in \operatorname{Hom}\left(\mathfrak{g}^{-1}, \mathfrak{g}^{0}\right)$ there exists a horizontal subspace $\widetilde{H}$ such that $f=f_{H \widetilde{H}}$. The map

$$
\partial: \operatorname{Hom}\left(\mathfrak{g}^{-1}, \mathfrak{g}^{0}\right) \rightarrow \operatorname{Hom}\left(\mathfrak{g}^{-1} \wedge \mathfrak{g}^{-1}, \mathfrak{g}^{-1}\right)
$$

defined by

$$
\partial f\left(v_{1}, v_{2}\right)=f\left(v_{1}\right) v_{2}-f\left(v_{2}\right) v_{1}=\left[f\left(v_{1}\right), v_{2}\right]+\left[v_{1}, f\left(v_{2}\right)\right]
$$


is called the Spencer operator ${ }^{1}$. By direct computations ([7, p. 42], [9, p. 317], or the proof of more general statement in Proposition 3.1 below) one obtains the following identity

$$
C_{\widetilde{H}}=C_{H}+\partial f_{H \widetilde{H}}
$$

Now fix a subspace

$$
\mathcal{N} \subset \operatorname{Hom}\left(\mathfrak{g}^{-1} \wedge \mathfrak{g}^{-1}, \mathfrak{g}^{-1}\right)
$$

complementary to $\operatorname{Im} \partial$, so that

$$
\operatorname{Hom}\left(\mathfrak{g}^{-1} \wedge \mathfrak{g}^{-1}, \mathfrak{g}^{-1}\right)=\operatorname{Im} \partial \oplus \mathcal{N} .
$$

Speaking informally, the subspace $\mathcal{N}$ defines the normalization conditions for the first prolongation. The first prolongation of $P^{0}$ is the following bundle $\left(P^{0}\right)^{(1)}$ over $P^{0}$ :

$$
\left(P^{0}\right)^{(1)}=\left\{(\lambda, H): \lambda \in P^{0}, H \text { is a horizontal subspace of } T_{\lambda} P^{0} \text { with } C_{H} \in \mathcal{N}\right\} .
$$

Alternatively,

$$
\left(P^{0}\right)^{(1)}=\left\{\left(\lambda, \varphi^{H}\right): \lambda \in P^{0}, H \text { is a horizontal subspace of } T_{\lambda} P^{0} \text { with } C_{H} \in \mathcal{N}\right\} .
$$

In other words, the fiber of $\left(P^{0}\right)^{(1)}$ over a point $\lambda \in P^{0}$ is the set of all horizontal subspaces $H$ of $T_{\lambda} P^{0}$ such that their structure functions satisfy the chosen normalization condition $\mathcal{N}$. Obviously, the fibers of $\left(P^{0}\right)^{(1)}$ are not empty, and if two horizontal subspaces $H, \tilde{H}$ belong to the fiber, then $f_{H \widetilde{H}} \in$ ker $\partial$. The subspace $\mathfrak{g}^{1}$ of $\operatorname{Hom}\left(\mathfrak{g}^{-1}, \mathfrak{g}^{0}\right)$ defined by

$$
\mathfrak{g}^{1} \stackrel{\text { def }}{=} \operatorname{ker} \partial \text {. }
$$

is called the first algebraic prolongation of $\mathfrak{g}^{0} \subset \mathfrak{g l}\left(\mathfrak{g}^{-1}\right)$. Note that it is absolutely not important that $\mathfrak{g}^{0}$ be a subalgebra of $\mathfrak{g l}\left(\mathfrak{g}^{-1}\right)$ : the first algebraic prolongation can be defined for a subspace of $\mathfrak{g l}\left(\mathfrak{g}^{-1}\right)$ (see the further generalization below).

If $\mathfrak{g}^{1}=0$ then the choice of the "normalization conditions" $\mathcal{N}$ determines an Ehresmann connection on $P^{0}$ and $P^{0}$ is endowed with a canonical frame. As an example consider a Riemannian structure. In this case $\mathfrak{g}^{0}=\mathfrak{s o}(n)$, where $n=\operatorname{dim} \mathfrak{g}^{-1}$, and it is easy to show that $\mathfrak{g}^{1}=0$. Moreover, $\operatorname{dim} \operatorname{Hom}\left(\mathfrak{g}^{-1} \wedge \mathfrak{g}^{-1}, \mathfrak{g}^{-1}\right)=\operatorname{dim} \operatorname{Hom}\left(\mathfrak{g}^{-1}, \mathfrak{g}^{0}\right)=\frac{n^{2}(n-1)}{2}$. Hence, $\operatorname{Im} \partial=\operatorname{Hom}\left(\mathfrak{g}^{-1} \wedge \mathfrak{g}^{-1}, \mathfrak{g}^{-1}\right)$ and the complement subspace $\mathcal{N}$ must be equal to 0 . So, in this case one gets the canonical Ehresmann connection with zero structure function (torsion), which is nothing but the Levi-Civita connection.

If $\mathfrak{g}^{1} \neq 0$, we continue the prolongation procedure by induction. Given a linear space $W$ denote by $\operatorname{Id}_{W}$ the identity map on $W$. The bundle $\left(P^{0}\right)^{(1)}$ is a frame bundle with the abelian structure group $G^{1}$ of all maps $A \in \mathrm{GL}\left(\mathfrak{g}^{-1} \oplus \mathfrak{g}^{0}\right)$ such that

$$
\begin{aligned}
& \left.A\right|_{\mathfrak{g}^{-1}}=\operatorname{Id}_{\mathfrak{g}^{-1}}+T, \\
& \left.A\right|_{\mathfrak{g}^{0}}=\operatorname{Id}_{\mathfrak{g}^{0}},
\end{aligned}
$$

\footnotetext{
${ }^{1}$ In [9] this operator is called the antisymmetrization operator, but we prefer to call it the Spencer operator, because, after certain intepretation of the spaces $\operatorname{Hom}\left(\mathfrak{g}^{-1}, \mathfrak{g}^{0}\right)$ and $\operatorname{Hom}\left(\mathfrak{g}^{-1} \wedge \mathfrak{g}^{-1}, \mathfrak{g}^{-1}\right)$, this operator can be identified with an appropriate $\delta$-operator introduced by Spencer in [8] for the study of overdetermined systems of partial differential equations. Indeed, since $\mathfrak{g}^{0}$ is a subspace of $\mathfrak{g l}\left(\mathfrak{g}^{-1}\right)$, the space Hom $\left(\mathfrak{g}^{-1}, \mathfrak{g}^{0}\right)$ can be seen as a subspace of the space of $\mathfrak{g}^{-1}$-valued one-forms on $\mathfrak{g}^{-1}$ with linear coefficients, while $\operatorname{Hom}\left(\mathfrak{g}^{-1} \wedge \mathfrak{g}^{-1}, \mathfrak{g}^{-1}\right)$ can be seen as the space of $\mathfrak{g}^{-1}$-valued two-forms on $\mathfrak{g}^{-1}$ with constant coefficients. Then the operator $\partial$ defined by (2.4) coincides with the restriction to $\operatorname{Hom}\left(\mathfrak{g}^{-1}, \mathfrak{g}^{0}\right)$ of the exterior differential acting between the above-mentioned spaces of one-forms and two-forms, i.e. with the corresponding Spencer $\delta$-operator.
} 
where $T \in \mathfrak{g}^{1}$. The right action $R_{A}$ of $A \in G_{1}$ on a fiber of $\left(P^{0}\right)^{(1)}$ is defined by the following rule: $R_{A}(\varphi)=\varphi \circ A$. Observe that $\mathfrak{g}^{1}$ is isomorphic to the Lie algebra of $G^{1}$.

Set $P^{1}=\left(P^{0}\right)^{(1)}$. The second prolongation $P^{2}$ of $P^{0}$ is by definition the first prolongation of the frame bundle $P^{1}, P^{2} \stackrel{\text { def }}{=}\left(P^{1}\right)^{(1)}$ and so on by induction: the $i$-th prolongation $P^{i}$ is the first prolongation of the frame bundle $P^{i-1}$.

Let us describe the structure group $G^{i}$ of the frame bundle $P^{i}$ over $P^{i-1}$ in more detail. For this one can define the Spencer operator and the first algebraic prolongation also for a subspace $W$ of $\operatorname{Hom}\left(\mathfrak{g}^{-1}, V\right)$, where $V$ is a linear space, which does not necessary coincide with $\mathfrak{g}^{-1}$ as before. In this case the Spencer operator is the operator from $\operatorname{Hom}\left(\mathfrak{g}^{-1}, W\right)$ to $\operatorname{Hom}\left(\mathfrak{g}^{-1} \wedge \mathfrak{g}^{-1}, V\right)$, defined by the same formulas, as in (2.4). The first prolongation $W^{(1)}$ of $W$ is the kernel of the Spencer operator. Note that by definition $\mathfrak{g}^{1}=\left(\mathfrak{g}^{0}\right)^{(1)}$. Then the $i$-th prolongation $\mathfrak{g}^{i}$ of $\mathfrak{g}^{0}$ is defined by the following recursive formula: $\mathfrak{g}^{i}=\left(\mathfrak{g}^{i-1}\right)^{(1)}$. Note that $\mathfrak{g}^{i} \subset \operatorname{Hom}\left(\mathfrak{g}^{-1}, \mathfrak{g}^{i-1}\right)$. By (2.5) and the definition of the Spencer operator the bundle $P^{i}$ is a frame bundle with the abelian structure group $G^{i}$ of all maps $A \in \operatorname{GL}\left(\bigoplus_{p=-1}^{i-1} \mathfrak{g}^{p}\right)$ such that

$$
\begin{aligned}
& \left.A\right|_{\mathfrak{g}^{-1}}=\operatorname{Id}_{\mathfrak{g}^{-1}}+T, \\
& \left.A\right|_{\bigoplus_{p=0}^{i-1} \mathfrak{g}^{p}}=\operatorname{Id}_{\bigoplus_{p=0}^{i-1} \mathfrak{g}^{p}},
\end{aligned}
$$

where $T \in \mathfrak{g}^{i}$. In particular, if $\mathfrak{g}^{l+1}=0$ for some $l \geq 0$, then the bundle $P^{l}$ is endowed with the canonical frame and we are done.

\section{Tanaka's first prolongation}

Now consider the general case. As before $P^{0}$ is a structure of constant type $\left(\mathfrak{m}, \mathfrak{g}^{0}\right)$. Let $\Pi_{0}: P^{0} \rightarrow M$ be the canonical projection. The filtration $\left\{D^{i}\right\}_{i<0}$ of $T M$ induces a filtration $\left\{D_{0}^{i}\right\}_{i \leq 0}$ of $T P^{0}$ as follows:

$$
\begin{aligned}
& D_{0}^{0}=\operatorname{ker}\left(\Pi_{0}\right)_{*}, \\
& D_{0}^{i}(\lambda)=\left\{v \in T_{\lambda} P^{0}:\left(\Pi_{0}\right)_{*} v \in D^{i}\left(\Pi_{0}(\lambda)\right)\right\} \quad \forall i<0 .
\end{aligned}
$$

We also set $D_{0}^{i}=0$ for all $i>0$. Note that $D_{0}^{0}(\lambda)$ is the tangent space at $\lambda$ to the fiber of $P^{0}$ and therefore can be identified with $\mathfrak{g}^{0}$. Denote by $I_{\lambda}: \mathfrak{g}^{0} \rightarrow D_{0}^{0}(\lambda)$ the identifying isomorphism.

Fix a point $\lambda \in P^{0}$ and let $\pi_{0}^{i}: D_{0}^{i}(\lambda) / D_{0}^{i+2}(\lambda) \rightarrow D_{0}^{i}(\lambda) / D_{0}^{i+1}(\lambda)$ be the canonical projection to the factor space. Note that $\Pi_{0_{*}}$ induces an isomorphism between the space $D_{0}^{i}(\lambda) / D_{0}^{i+1}(\lambda)$ and the space $D^{i}\left(\Pi_{0}(\lambda)\right) / D^{i+1}\left(\Pi_{0}(\lambda)\right)$ for any $i<0$. We denote this isomorphism by $\Pi_{0}^{i}$. The fiber of the bundle $P^{0}$ over a point $x \in M$ is a subset of the set of all maps

$$
\varphi \in \bigoplus_{i<0} \operatorname{Hom}\left(\mathfrak{g}^{i}, D^{i}(x) / D^{i+1}(x)\right)
$$

which are isomorphisms of the graded Lie algebras $\mathfrak{m}=\bigoplus_{i<0} \mathfrak{g}^{i}$ and $\bigoplus_{i<0} D^{i}(x) / D^{i+1}(x)$. We are going to construct a new bundle $P^{1}$ over the bundle $P^{0}$ such that the fiber of $P^{1}$ over a point $\lambda=(x, \varphi) \in P^{0}$ will be a certain subset of the set of all maps

$$
\hat{\varphi} \in \bigoplus_{i \leq 0} \operatorname{Hom}\left(\mathfrak{g}^{i}, D_{0}^{i}(\lambda) / D_{0}^{i+2}(\lambda)\right)
$$


such that

$$
\begin{aligned}
& \left.\varphi\right|_{\mathfrak{g}^{i}}=\left.\Pi_{0}^{i} \circ \pi_{0}^{i} \circ \hat{\varphi}\right|_{\mathfrak{g}^{i}} \quad \forall i<0, \\
& \left.\hat{\varphi}\right|_{\mathfrak{g}^{0}}=I_{\lambda}
\end{aligned}
$$

For this fix again a point $\lambda=(x, \varphi) \in P^{0}$. For any $i<0$ choose a subspace $H^{i} \subset D_{0}^{i}(\lambda) / D_{0}^{i+2}(\lambda)$, which is a complement of $D_{0}^{i+1}(\lambda) / D_{0}^{i+2}(\lambda)$ to $D_{0}^{i}(\lambda) / D_{0}^{i+2}(\lambda)$ :

$$
D_{0}^{i}(\lambda) / D_{0}^{i+2}(\lambda)=D_{0}^{i+1}(\lambda) / D_{0}^{i+2}(\lambda) \oplus H^{i} .
$$

Then the map $\left.\Pi_{0}^{i} \circ \pi_{0}^{i}\right|_{H^{i}}$ defines an isomorphism between $H^{i}$ and $D^{i}\left(\Pi_{0}(\lambda)\right) / D^{i+1}\left(\Pi_{0}(\lambda)\right)$. So, once a tuple of subspaces $\mathcal{H}=\left\{H^{i}\right\}_{i<0}$ is chosen, one can define a map

$$
\varphi^{\mathcal{H}} \in \bigoplus_{i \leq 0} \operatorname{Hom}\left(\mathfrak{g}^{i}, D_{0}^{i}(\lambda) / D_{0}^{i+2}(\lambda)\right)
$$

as follows

$$
\left.\varphi^{\mathcal{H}}\right|_{\mathfrak{g}^{i}}= \begin{cases}\left.\left(\left.\Pi_{0}^{i} \circ \pi_{0}^{i}\right|_{H^{i}}\right)^{-1} \circ \varphi\right|_{\mathfrak{g}^{i}} & \text { if } i<0, \\ I_{\lambda} & \text { if } i=0 .\end{cases}
$$

Clearly $\hat{\varphi}=\varphi^{\mathcal{H}}$ satisfies (3.1). Tuples of subspaces $\mathcal{H}=\left\{H^{i}\right\}_{i<0}$ satisfying (3.2) play here the same role as horizontal subspaces in the prolongation of the usual $G$-structures. Can we choose a tuple $\left\{H^{i}\right\}_{i<0}$ in a canonical way? For this, by analogy with the prolongation of $G$-structure, we introduce a "partial soldering form" of the bundle $P^{0}$ and the structure function of a tuple $\mathcal{H}$. The soldering form of $P^{0}$ is a tuple $\Omega_{0}=\left\{\omega_{0}^{i}\right\}_{i<0}$, where $\omega_{0}^{i}$ is a $\mathfrak{g}^{i}$-valued linear form on $D_{0}^{i}(\lambda)$ defined by

$$
\omega_{0}^{i}(Y)=\varphi^{-1}\left(\left(\left(\Pi_{0}\right)_{*}(Y)\right)_{i}\right),
$$

where $\left(\left(\Pi_{0}\right)_{*}(Y)\right)_{i}$ is the equivalence class of $\left(\Pi_{0}\right)_{*}(Y)$ in $D^{i}(x) / D^{i+1}(x)$. Observe that $D_{0}^{i+1}(\lambda)$ $=\operatorname{ker} \omega_{0}^{i}$. Thus the form $\omega_{0}^{i}$ induces the $\mathfrak{g}^{i}$-valued form $\bar{\omega}_{0}^{i}$ on $D_{0}^{i}(\lambda) / D_{0}^{i+1}(\lambda)$. The structure function $C_{\mathcal{H}}^{0}$ of the tuple $\mathcal{H}=\left\{H^{i}\right\}_{i<0}$ is the element of the space

$$
\mathcal{A}_{0}=\left(\bigoplus_{i=-\mu}^{-2} \operatorname{Hom}\left(\mathfrak{g}^{-1} \otimes \mathfrak{g}^{i}, \mathfrak{g}^{i}\right)\right) \oplus \operatorname{Hom}\left(\mathfrak{g}^{-1} \wedge \mathfrak{g}^{-1}, \mathfrak{g}^{-1}\right)
$$

defined as follows. Let $\operatorname{pr}_{i}^{\mathcal{H}}$ be the projection of $D_{0}^{i}(\lambda) / D_{0}^{i+2}(\lambda)$ to $D_{0}^{i+1}(\lambda) / D_{0}^{i+2}(\lambda)$ parallel to $H^{i}$ (or corresponding to the splitting (3.2)). Given vectors $v_{1} \in \mathfrak{g}^{-1}$ and $v_{2} \in \mathfrak{g}^{i}$, take two vector fields $Y_{1}$ and $Y_{2}$ in a neighborhood of $\lambda$ in $P^{0}$ such that $Y_{1}$ is a section of $D_{0}^{-1}, Y_{2}$ is a section of $D_{0}^{i}$, and

$$
\begin{aligned}
& \omega_{0}^{-1}\left(Y_{1}\right) \equiv v_{1}, \quad \omega_{0}^{i}\left(Y_{2}\right) \equiv v_{2}, \\
& Y_{1}(\lambda)=\varphi^{\mathcal{H}}\left(v_{1}\right), \quad Y_{2}(\lambda) \equiv \varphi^{\mathcal{H}}\left(v_{2}\right) \bmod D_{0}^{i+2}(\lambda) .
\end{aligned}
$$

Then set

$$
C_{\mathcal{H}}^{0}\left(v_{1}, v_{2}\right) \stackrel{\text { def }}{=} \bar{\omega}_{0}^{i}\left(\operatorname{pr}_{i-1}^{\mathcal{H}}\left(\left[Y_{1}, Y_{2}\right](\lambda)\right)\right) .
$$

In the above formula we take the equivalence class of the vector $\left[Y_{1}, Y_{2}\right](\lambda)$ in $D_{0}^{i-1}(\lambda) / D_{0}^{i+1}(\lambda)$ and then apply $\operatorname{pr}_{i-1}^{\mathcal{H}}$. 
One must show that $C_{\mathcal{H}}^{0}\left(v_{1}, v_{2}\right)$ does not depend on the choice of vector fields $Y_{1}$ and $Y_{2}$, satisfying (3.4). Indeed, assume that $\widetilde{Y}_{1}$ and $\widetilde{Y}_{2}$ are another pair of vector fields in a neighborhood of $\lambda$ in $P^{0}$ such that $\widetilde{Y}_{1}$ is a section of $D_{0}^{-1}, \widetilde{Y}_{2}$ is a section of $D_{0}^{i}$, and they satisfy (3.4) with $Y_{1}, Y_{2}$ replaced by $\tilde{Y}_{1}, \widetilde{Y}_{1}$. Then

$$
\tilde{Y}_{1}=Y_{1}+Z_{1}, \quad \tilde{Y}_{2}=Y_{2}+Z_{2},
$$

where $Z_{1}$ is a section of the distribution $D_{0}^{0}$ such that $Z_{1}(\lambda)=0$ and $Z_{2}$ is a section of the distribution $D_{0}^{i+1}$ such that $Z_{2}(\lambda) \in D_{0}^{i+2}(\lambda)$. It follows that $\left[Y_{1}, Z_{2}\right](\lambda) \in D_{0}^{i+1}(\lambda)$ and $\left[Y_{2}, Z_{1}\right](\lambda) \in D_{0}^{i+1}(\lambda)$. This together with the fact that $\left[Z_{1}, Z_{2}\right]$ is a section of $D_{0}^{i+1}$ imply that

$$
\left[\tilde{Y}_{1}, \widetilde{Y}_{2}\right](\lambda) \equiv\left[Y_{1}, Y_{2}\right] \bmod D_{0}^{i+1}(\lambda) .
$$

From (3.5) we see that the structure function is independent of the choice of vector fields $Y_{1}$ and $Y_{2}$.

We now take another tuple $\widetilde{\mathcal{H}}=\left\{\widetilde{H}^{i}\right\}_{i<0}$ such that

$$
D_{0}^{i}(\lambda) / D_{0}^{i+2}(\lambda)=D_{0}^{i+1}(\lambda) / D_{0}^{i+2}(\lambda) \oplus \widetilde{H}^{i}
$$

and consider how the structure functions $C_{\mathcal{H}}^{1}$ and $C_{\widetilde{\mathcal{H}}}^{1}$ are related. By construction, for any vector $v \in \mathfrak{g}^{i}$ the vector $\varphi^{\widetilde{\mathcal{H}}}(v)-\varphi^{\mathcal{H}}(v)$ belongs to $D_{0}^{i+1}(\lambda) / D_{0}^{i+2}(\lambda)$. Let

$$
f_{\mathcal{H} \widetilde{\mathcal{H}}}(v) \stackrel{\text { def }}{=} \begin{cases}\bar{\omega}_{0}^{i+1}\left(\varphi^{\tilde{\mathcal{H}}}(v)-\varphi^{\mathcal{H}}(v)\right) & \text { if } v \in \mathfrak{g}^{i} \text { with } i<-1, \\ I_{\lambda}^{-1}\left(\varphi^{\widetilde{\mathcal{H}}}(v)-\varphi^{\mathcal{H}}(v)\right) & \text { if } v \in \mathfrak{g}^{-1}\end{cases}
$$

Then $f_{\mathcal{H} \widetilde{\mathcal{H}}} \in \bigoplus_{i<0} \operatorname{Hom}\left(\mathfrak{g}^{i}, \mathfrak{g}^{i+1}\right)$. Conversely, it is clear that for any $f \in \bigoplus_{i<0} \operatorname{Hom}\left(\mathfrak{g}^{i}, \mathfrak{g}^{i+1}\right)$ there exists a tuple $\widetilde{\mathcal{H}}=\left\{\widetilde{H}^{i}\right\}_{i<0}$, satisfying (3.7), such that $f=f_{\mathcal{H} \widetilde{\mathcal{H}}}$.

Further, let $\mathcal{A}_{0}$ be as in (3.3) and define a map

$$
\partial_{0}: \bigoplus_{i<0} \operatorname{Hom}\left(\mathfrak{g}^{i}, \mathfrak{g}^{i+1}\right) \rightarrow \mathcal{A}_{0}
$$

by

$$
\partial_{0} f\left(v_{1}, v_{2}\right)=\left[f\left(v_{1}\right), v_{2}\right]+\left[v_{1}, f\left(v_{2}\right)\right]-f\left(\left[v_{1}, v_{2}\right]\right)
$$

where the brackets $[$,$] are as in the Lie algebra \mathfrak{m} \oplus \mathfrak{g}^{0}$. The map $\partial_{0}$ coincides with the Spencer operator (2.4) in the case of $G$-structures. Therefore it is called the generalized Spencer operator for the first prolongation.

Proposition 3.1. The following identity holds

$$
C_{\widetilde{\mathcal{H}}}^{0}=C_{\mathcal{H}}^{0}+\partial_{0} f_{\mathcal{H} \widetilde{\mathcal{H}}}
$$

Proof. Fix vectors $v_{1} \in \mathfrak{g}^{-1}$ and $v_{2} \in \mathfrak{g}^{i}$ and let $Y_{1}$ and $Y_{2}$ be two vector fields in a neighborhood of $\lambda$ satisfying (3.4). Take two vector fields $\widetilde{Y}_{1}$ and $\widetilde{Y}_{2}$ in a neighborhood of $\lambda$ in $P^{0}$ such that $\widetilde{Y}_{1}$ is a section of $D_{0}^{-1}, \widetilde{Y}_{2}$ a section of $D_{0}^{i}$, and

$$
\begin{aligned}
& \omega_{0}^{-1}\left(\widetilde{Y}_{1}\right) \equiv v_{1}, \quad \omega_{0}^{i}\left(\widetilde{Y}_{2}\right) \equiv v_{2}, \\
& \widetilde{Y}_{1}(\lambda)=\varphi^{\widetilde{\mathcal{H}}}\left(v_{1}\right), \quad \widetilde{Y}_{2}(\lambda) \equiv \varphi^{\widetilde{\mathcal{H}}}\left(v_{2}\right) \bmod D_{0}^{i+2}(\lambda) .
\end{aligned}
$$


Further, assume that vector fields $Z_{1}$ and $Z_{2}$ are defined as in (3.6). Then $Z_{1}$ is a section of $D_{0}^{0}$ and $Z_{2}$ is a section of $D_{0}^{i+1}$ such that

$$
\begin{aligned}
& Z_{1}(\lambda)=I_{\lambda}\left(f_{\mathcal{H} \widetilde{\mathcal{H}}}\left(v_{1}\right)\right), \\
& f_{\mathcal{H} \widetilde{\mathcal{H}}}\left(v_{2}\right)= \begin{cases}\bar{\omega}_{0}^{i+1}\left(Z_{2}(\lambda)\right) & \text { if } v \in \mathfrak{g}^{i}, i<-1, \\
I_{\lambda}^{-1}\left(Z_{2}(\lambda)\right) & \text { if } v \in \mathfrak{g}^{-1}\end{cases}
\end{aligned}
$$

Hence $\left[Z_{1}, Y_{2}\right]$ and $\left[Y_{1}, Z_{2}\right]$ are sections of $D_{0}^{i}$, while $\left[Z_{1}, Z_{2}\right]$ is a section of $D_{0}^{i+1}$. This implies that

$$
\bar{\omega}_{0}^{i}\left(\operatorname{pr}_{i-1}^{\widetilde{\mathcal{H}}}\left(\left[\widetilde{Y}_{1}, \widetilde{Y}_{2}\right](\lambda)\right)\right)=\bar{\omega}_{0}^{i}\left(\operatorname{pr}_{i-1}^{\widetilde{\mathcal{H}}}\left(\left[Y_{1}, Y_{2}\right](\lambda)\right)\right)+\bar{\omega}_{0}^{i}\left(\left[Z_{1}, Y_{2}\right]\right)+\bar{\omega}_{0}^{i}\left(\left[Y_{1}, Z_{2}\right]\right) .
$$

Further, directly from the definitions of $f_{\mathcal{H} \tilde{\mathcal{H}}}, \operatorname{pr}_{i-1}^{\mathcal{H}}$, and $\operatorname{pr}_{i-1}^{\widetilde{\mathcal{H}}}$ it follows that

$$
\bar{\omega}_{0}^{i}\left(\operatorname{pr}_{i-1}^{\widetilde{\mathcal{H}}}(w)\right)=\bar{\omega}_{0}^{i}\left(\operatorname{pr}_{i-1}^{\mathcal{H}}(w)\right)-f_{\mathcal{H} \widetilde{\mathcal{H}}}\left(\bar{\omega}_{0}^{i-1}(w)\right) \quad \forall w \in D_{0}^{i-1}(\lambda) / D_{0}^{i+1}(\lambda) .
$$

Besides, from the definition of the soldering form, the fact that $\varphi$ is an isomorphism of the Lie algebras $\mathfrak{m}$ and $\mathfrak{m}(x)=\bigoplus_{i<0} D^{i}(x) / D^{i+1}(x)$, and relations (3.10) for $i<-1$ it follows that

$$
\bar{\omega}_{0}^{i}\left(\left[Y_{1}, Z_{2}\right]\right)=\left[v_{1}, f_{\mathcal{H} \widetilde{\mathcal{H}}}\left(v_{2}\right)\right] \quad \forall i<-1 .
$$

Taking into account (3.4) we get

$$
\bar{\omega}_{0}^{i-1}\left(\left[Y_{1}, Y_{2}\right]\right)=\left[v_{1}, v_{2}\right] .
$$

Finally, from (3.9) and (3.10) for $i=-1$, and the definition of the action of $G^{0}$ on $P^{0}$ it follows that identity (3.13) holds also for $i=-1$, and that

$$
\bar{\omega}_{0}^{i}\left(\left[Z_{1}, Y_{2}\right]\right)=\left[f_{\mathcal{H} \widetilde{\mathcal{H}}}\left(v_{1}\right), v_{2}\right] .
$$

Substituting (3.12)-(3.15) into (3.11) we get (3.8).

Now we proceed as in the case of $G$-structures. Fix a subspace

$$
\mathcal{N}_{0} \subset \mathcal{A}_{0}
$$

which is complementary to $\operatorname{Im} \partial_{0}$,

$$
\mathcal{A}_{0}=\operatorname{Im} \partial_{0} \oplus \mathcal{N}_{0}
$$

As for $G$-structures, the subspace $\mathcal{N}_{0}$ defines the normalization conditions for the first prolongation. Then from the splitting (3.16) it follows trivially that there exists a tuple $\mathcal{H}=\left\{H^{i}\right\}_{i<0}$ such that

$$
C_{\mathcal{H}}^{0} \in \mathcal{N}_{0}
$$

A tuple $\widetilde{\mathcal{H}}=\left\{\widetilde{H}^{i}\right\}_{i<0}$ satisfies $C_{\widetilde{\mathcal{H}}}^{0} \in \mathcal{N}_{0}$ if and only if $f_{\mathcal{H} \widetilde{\mathcal{H}}} \in$ ker $\partial_{0}$. In particular if ker $\partial_{0}=0$ then the tuple $\mathcal{H}$ is fixed uniquely by condition (3.17). Let

$$
\mathfrak{g}^{1} \stackrel{\text { def }}{=} \operatorname{ker} \partial_{0} \text {. }
$$


The space $\mathfrak{g}^{1}$ is called the first algebraic prolongation of the algebra $\mathfrak{m} \oplus \mathfrak{g}^{0}$. Here we consider $\mathfrak{g}^{1}$ as an abelian Lie algebra. Note that the fact that the symbol $\mathfrak{m}$ is fundamental (that is, $\mathfrak{g}^{-1}$ generates the whole $\mathfrak{m}$ ) implies that

$$
\mathfrak{g}^{1}=\left\{f \in \bigoplus_{i<0} \operatorname{Hom}\left(\mathfrak{g}^{i}, \mathfrak{g}^{i+1}\right): f\left(\left[v_{1}, v_{2}\right]\right)=\left[f\left(v_{1}\right), v_{2}\right]+\left[v_{1}, f\left(v_{2}\right)\right] \forall v_{1}, v_{2} \in \mathfrak{m}\right\} .
$$

The first (geometric) prolongation of the bundle $P^{0}$ is the bundle $P^{1}$ over $P^{0}$ defined by

$$
P^{1}=\left\{(\lambda, \mathcal{H}): \lambda \in P^{0}, C_{\mathcal{H}}^{0} \in \mathcal{N}_{0}\right\} .
$$

Equivalently,

$$
P^{1}=\left\{\left(\lambda, \varphi^{\mathcal{H}}\right): \lambda \in P^{0}, C_{\mathcal{H}}^{0} \in \mathcal{N}_{0}\right\} .
$$

It is a principal bundle with the abelian structure group $G^{1}$ of all maps $A \in \bigoplus_{i<1} \operatorname{Hom}\left(\mathfrak{g}^{i}, \mathfrak{g}^{i} \oplus \mathfrak{g}^{i+1}\right)$ such that

$$
\begin{aligned}
\left.A\right|_{\mathfrak{g}^{i}} & =\operatorname{Id}_{\mathfrak{g}^{i}}+T_{i}, \quad i<0, \\
\left.A\right|_{\mathfrak{g}^{0}} & =\operatorname{Id}_{\mathfrak{g}^{0}},
\end{aligned}
$$

where $T_{i} \in \operatorname{Hom}\left(\mathfrak{g}^{i}, \mathfrak{g}^{i+1}\right)$ and $\left(T_{-\mu}, \ldots, T_{-1}\right) \in \mathfrak{g}^{1}$. The right action $R_{A}^{1}$ of $A \in G^{1}$ on a fiber of $P^{1}$ is defined by $R_{A}^{1}\left(\varphi^{\mathcal{H}}\right)=\varphi^{\mathcal{H}} \circ A$. Note that $G^{1}$ is an abelian group of dimension equal to $\operatorname{dim} \mathfrak{g}^{1}$.

\section{Higher order Tanaka's prolongations}

More generally, define the $k$-th algebraic prolongation $\mathfrak{g}^{k}$ of the algebra $\mathfrak{m} \oplus \mathfrak{g}^{0}$ by induction for any $k \in \mathbb{N}$. Assume that spaces $\mathfrak{g}^{l} \subset \bigoplus_{i<0} \operatorname{Hom}\left(\mathfrak{g}^{i}, \mathfrak{g}^{i+l}\right)$ are defined for all $0<l<k$. Set

$$
[f, v]=-[v, f]=f(v) \quad \forall f \in \mathfrak{g}^{l}, 0 \leq l<k, \text { and } v \in \mathfrak{m} .
$$

Then let

$$
\mathfrak{g}^{k} \stackrel{\text { def }}{=}\left\{f \in \bigoplus_{i<0} \operatorname{Hom}\left(\mathfrak{g}^{i}, \mathfrak{g}^{i+k}\right): f\left(\left[v_{1}, v_{2}\right]\right)=\left[f\left(v_{1}\right), v_{2}\right]+\left[v_{1}, f\left(v_{2}\right)\right] \forall v_{1}, v_{2} \in \mathfrak{m}\right\} .
$$

Directly from this definition and the fact that $\mathfrak{m}$ is fundamental (that is, it is generated by $\mathfrak{g}^{-1}$ ) it follows that if $f \in \mathfrak{g}^{k}$ satisfies $\left.f\right|_{\mathfrak{g}^{-1}}=0$, then $f=0$. The space $\bigoplus_{i \in Z} \mathfrak{g}^{i}$ can be naturally endowed with the structure of a graded Lie algebra. The brackets of two elements from $\mathfrak{m}$ are as in $\mathfrak{m}$. The brackets of an element with non-negative weight and an element from $\mathfrak{m}$ are already defined by (4.1). It only remains to define the brackets $\left[f_{1}, f_{2}\right]$ for $f_{1} \in \mathfrak{g}^{k}, f_{2} \in \mathfrak{g}^{l}$ with $k, l \geq 0$. The definition is inductive with respect to $k$ and $l$ : if $k=l=0$ then the bracket $\left[f_{1}, f_{2}\right]$ is as in $\mathfrak{g}^{0}$. Assume that $\left[f_{1}, f_{2}\right]$ is defined for all $f_{1} \in \mathfrak{g}^{k}, f_{2} \in \mathfrak{g}^{l}$ such that a pair $(k, l)$ belongs to the set

$$
\{(k, l): 0 \leq k \leq \bar{k}, 0 \leq l \leq \bar{l}\} \backslash\{(\bar{k}, \bar{l})\} .
$$

Then define $\left[f_{1}, f_{2}\right]$ for $f_{1} \in \mathfrak{g}^{\bar{k}}, f_{2} \in \mathfrak{g}^{\bar{l}}$ to be the element of $\bigoplus_{i<0} \operatorname{Hom}\left(\mathfrak{g}^{i}, \mathfrak{g}^{i+\bar{k}+\bar{l}}\right)$ given by

$$
\left[f_{1}, f_{2}\right] v \stackrel{\text { def }}{=}\left[f_{1}(v), f_{2}\right]+\left[f_{1}, f_{2}(v)\right] \quad \forall v \in \mathfrak{m} .
$$


It is easy to see that $\left[f_{1}, f_{2}\right] \in \mathfrak{g}^{k+l}$ and that $\bigoplus_{i \in Z} \mathfrak{g}^{i}$ with bracket product defined as above is a graded Lie algebra. As a matter of fact $[10, \S 5]$ this graded Lie algebra satisfies Properties 1-3 from Subsection 1.4. That is it is a realization of the algebraic universal prolongation $\mathfrak{g}\left(\mathfrak{m}, \mathfrak{g}^{0}\right)$ of the algebra $\mathfrak{m} \oplus \mathfrak{g}^{0}$.

Now we are ready to construct the higher order geometric prolongations of the bundle $P^{0}$ by induction. Assume that all $l$-th order prolongations $P^{l}$ are constructed for $0 \leq l \leq k$. We also set $P^{-1}=M$. We will not specify what the bundles $P^{l}$ are exactly. As in the case of the first prolongation $P^{1}$, their construction depends on the choice of normalization conditions on each step. But we will point out those properties of these bundles that we need in order to construct the $(k+1)$-st order prolongation $P^{k+1}$. Here are these properties:

1. $P^{l}$ is a principal bundle over $P^{l-1}$ with an abelian structure group $G^{l}$ of dimension equal to $\operatorname{dim} \mathfrak{g}^{l}$ and with the canonical projection $\Pi_{l}$.

2. The tangent bundle $T P^{l}$ is endowed with the filtration $\left\{D_{l}^{i}\right\}$ as follows: For $l=-1$ it coincides with the initial filtration $\left\{D^{i}\right\}_{i<0}$ and for $l \geq 0$ we get by induction

$$
\begin{aligned}
& D_{l}^{l}=\operatorname{ker}\left(\Pi_{l}\right)_{*}, \\
& D_{l}^{i}\left(\lambda_{l}\right)=\left\{v \in T_{\lambda} P^{l}:\left(\Pi_{l}\right)_{*} v \in D_{l-1}^{i}\left(\Pi_{l}\left(\lambda_{l}\right)\right)\right\} \quad \forall i<l .
\end{aligned}
$$

The subspaces $D_{l}^{l}\left(\lambda_{l}\right)$, as the tangent spaces to the fibers of $P^{l}$, are canonically identified with $\mathfrak{g}^{l}$. Denote by $I_{\lambda_{l}}: \mathfrak{g}^{l} \rightarrow D_{l}^{l}\left(\lambda_{l}\right)$ the identifying isomorphism.

3. The fiber of $P^{l}, 0 \leq l \leq k$, over a point $\lambda_{l-1} \in P^{l-1}$ will be a certain subset of the set of all maps from

$$
\bigoplus_{i<l} \operatorname{Hom}\left(\mathfrak{g}^{i}, D_{l-1}^{i}\left(\lambda_{l-1}\right) / D_{l-1}^{i+l+1}\left(\lambda_{l-1}\right)\right) .
$$

If $l>0$ and $\lambda_{l}=\left(\lambda_{l-1}, \varphi_{l}\right) \in P^{l}$, then $\left.\varphi_{l}\right|_{\mathfrak{g}^{l-1}}$ coincides with the identification of $\mathfrak{g}^{l-1}$ with $D_{l-1}^{l-1}\left(\lambda_{l-1}\right)$ and the restrictions $\left.\varphi_{l}\right|_{\mathfrak{g}^{i}}$ with $i \geq 0$ are the same for all $\lambda_{l}$ from the same fiber.

4. Assume that $0<l \leq k, \lambda_{l-1}=\left(\lambda_{l-2}, \varphi_{l-1}\right) \in P^{l-1}$ and $\lambda_{l}=\left(\lambda_{l-1}, \varphi_{l}\right) \in P^{l}$. The maps $\varphi_{l-1}$ and $\varphi_{l}$ are related as follows: if

$$
\pi_{l}^{i}: D_{l}^{i}\left(\lambda_{l}\right) / D_{l}^{i+l+2}\left(\lambda_{l}\right) \rightarrow D_{l}^{i}\left(\lambda_{l}\right) / D_{l}^{i+l+1}\left(\lambda_{l}\right)
$$

are the canonical projections to a factor space and

$$
\Pi_{l}^{i}: D_{l}^{i}\left(\lambda_{l}\right) / D_{l}^{i+l+1}\left(\lambda_{l}\right) \rightarrow D_{l-1}^{i}\left(\Pi_{l}\left(\lambda_{l}\right)\right) / D_{l-1}^{i+l+1}\left(\Pi_{l}\left(\lambda_{l}\right)\right)
$$

are the canonical maps induced by $\left(\Pi_{l}\right)_{*}$, then

$$
\forall i<\left.l \quad \varphi_{l-1}\right|_{\mathfrak{g}^{i}}=\left.\Pi_{l-1}^{i} \circ \pi_{l-1}^{i} \circ \varphi_{l}\right|_{\mathfrak{g}^{i}} .
$$

Note that the maps $\Pi_{l}^{i}$ are isomorphisms for $i<0$ and the maps $\pi_{l}^{i}$ are identities for $i \geq 0$ (we set $D_{l}^{i}=0$ for $i>l$ ).

Now we are ready to construct the $(k+1)$-st order Tanaka geometric prolongation. Fix a point $\lambda_{k} \in P^{k}$ and assume that $\lambda_{k}=\left(\lambda_{k-1}, \varphi_{k}\right)$, where

$$
\varphi_{k} \in \bigoplus_{i<k} \operatorname{Hom}\left(\mathfrak{g}^{i}, D_{k-1}^{i}\left(\lambda_{k-1}\right) / D_{k-1}^{i+k+1}\left(\lambda_{k-1}\right)\right) .
$$

Let $\mathcal{H}_{k}=\left\{H_{k}^{i}\right\}_{i<k}$ be the tuple of spaces such that $H_{k}^{i}=\varphi_{k}\left(\mathfrak{g}^{i}\right)$. Take a tuple $\mathcal{H}_{k+1}=\left\{H_{k+1}^{i}\right\}_{i<k}$ of linear spaces such that 
1. for $i<0$ the space $H_{k+1}^{i}$ is a complement of $D_{k}^{i+k+1}\left(\lambda_{k}\right) / D_{k}^{i+k+2}\left(\lambda_{k}\right)$ in $\left(\Pi_{k}^{i} \circ \pi_{k}^{i}\right)^{-1}\left(H_{k}^{i}\right) \subset$ $D_{k}^{i}\left(\lambda_{k}\right) / D_{k}^{i+k+2}\left(\lambda_{k}\right)$,

$$
\left(\Pi_{k}^{i} \circ \pi_{k}^{i}\right)^{-1}\left(H_{k}^{i}\right)=D_{k}^{i+k+1}\left(\lambda_{k}\right) / D_{k}^{i+k+2}\left(\lambda_{k}\right) \oplus H_{k+1}^{i} ;
$$

2. for $0 \leq i<k$ the space $H_{k+1}^{i}$ is a complement of $D_{k}^{k}\left(\lambda_{k}\right)$ in $\left(\Pi_{k}^{i}\right)^{-1}\left(H_{k}^{i}\right)$,

$$
\left(\Pi_{k}^{i}\right)^{-1}\left(H_{k}^{i}\right)=D_{k}^{k}\left(\lambda_{k}\right) \oplus H_{k+1}^{i} .
$$

Here the maps $\pi_{k}^{i}$ and $\Pi_{k}^{i}$ are defined as in (4.4) and (4.5) with $l=k$.

Since $D_{k}^{i+k+1}\left(\lambda_{k}\right) / D_{k}^{i+k+2}\left(\lambda_{k}\right)=\operatorname{ker} \pi_{k}^{i}$ and $\Pi_{k}^{i}$ is an isomorphism for $i<0$, the map $\Pi_{k}^{i} \circ$ $\left.\pi_{k}^{i}\right|_{H_{k+1}^{i}}$ defines an isomorphism between $H_{k+1}^{i}$ and $H_{k}^{i}$ for $i<0$. Additionally, by (4.7) the map $\left.\left(\Pi_{l}\right)_{*}\right|_{H_{k+1}^{i}}$ defines an isomorphism between $H_{k+1}^{i}$ and $H_{k}^{i}$ for $0 \leq i<k$. So, once a tuple of subspaces $\mathcal{H}_{k+1}=\left\{H_{k+1}^{i}\right\}_{i<k}$, satisfying (4.6) and (4.7), is chosen, one can define a map

$$
\varphi^{\mathcal{H}_{k+1}} \in \bigoplus_{i \leq k} \operatorname{Hom}\left(\mathfrak{g}^{i}, D_{k}^{i}\left(\lambda_{k}\right) / D_{k}^{i+k+2}\left(\lambda_{k}\right)\right)
$$

as follows

$$
\left.\varphi^{\mathcal{H}_{k+1}}\right|_{\mathfrak{g}^{i}}= \begin{cases}\left.\left(\left.\Pi_{k}^{i} \circ \pi_{k}^{i}\right|_{H_{k+1}^{i}}\right)^{-1} \circ \varphi_{k}\right|_{\mathfrak{g}^{i}} & \text { if } i<0, \\ \left.\left(\left.\left(\Pi_{l}\right)_{*}\right|_{H_{k+1}^{i}}\right)^{-1} \circ \varphi_{k}\right|_{\mathfrak{g}^{i}} & \text { if } 0 \leq i<k, \\ I_{\lambda_{k}} & \text { if } i=k .\end{cases}
$$

Can we choose a tuple or a subset of tuples $\mathcal{H}_{k}$ in a canonical way? To answer this question, by analogy with Sections 2 and 3, we introduce a "partial soldering form" of the bundle $P^{k}$ and the structure function of a tuple $\mathcal{H}_{k+1}$. The soldering form of $P^{k}$ is a tuple $\Omega_{k}=\left\{\omega_{k}^{i}\right\}_{i<k}$, where $\omega_{k}^{i}$ is a $\mathfrak{g}^{i}$-valued linear form on $D_{k}^{i}\left(\lambda_{k}\right)$ defined by

$$
\omega_{k}^{i}(Y)=\varphi_{k}^{-1}\left(\left(\left(\Pi_{k}\right)_{*}(Y)\right)_{i}\right) .
$$

Here $\left(\left(\Pi_{k}\right)_{*}(Y)\right)_{i}$ is the equivalence class of $\left(\Pi_{k}\right)_{*}(Y)$ in $D_{k-1}^{i}\left(\lambda_{k-1}\right) / D_{k-1}^{i+k+1}\left(\lambda_{k-1}\right)$. By construction it follows immediately that $D_{k}^{i+1}\left(\lambda_{k}\right)=\operatorname{ker} \omega_{k}^{i}$. So, the form $\omega_{k}^{i}$ induces the $\mathfrak{g}^{i}$-valued form $\bar{\omega}_{k}^{i}$ on $D_{k}^{i}\left(\lambda_{k}\right) / D_{k}^{i+1}\left(\lambda_{k}\right)$.

The structure function $C_{\mathcal{H}_{k+1}}^{k}$ of a tuple $\mathcal{H}_{k+1}$ is the element of the space

$$
\begin{aligned}
\mathcal{A}_{k}= & \left(\bigoplus_{i=-\mu}^{-2} \operatorname{Hom}\left(\mathfrak{g}^{-1} \otimes \mathfrak{g}^{i}, \mathfrak{g}^{i+k}\right)\right) \oplus \operatorname{Hom}\left(\mathfrak{g}^{-1} \wedge \mathfrak{g}^{-1}, \mathfrak{g}^{k-1}\right) \\
& \oplus\left(\bigoplus_{i=0}^{k-1} \operatorname{Hom}\left(\mathfrak{g}^{-1} \otimes \mathfrak{g}^{i}, \mathfrak{g}^{k-1}\right)\right)
\end{aligned}
$$

defined as follows: Let $\pi_{l}^{i, s}: D_{l}^{i}\left(\lambda_{l}\right) / D_{l}^{i+l+2}\left(\lambda_{l}\right) \rightarrow D_{l}^{i}\left(\lambda_{l}\right) / D_{l}^{i+l+2-s}\left(\lambda_{l}\right)$ be the canonical projection to a factor space, where $-1 \leq l \leq k, i \leq l$. Here, as before, we assume that $D_{l}^{i}=0$ for $i>l$. Note that the previously defined $\pi_{l}^{i}$ coincides with $\pi_{l}^{i, 1}$. By construction, one has the following two relations

$$
\begin{aligned}
& D_{k}^{i}\left(\lambda_{k}\right) / D_{k}^{i+k+2}\left(\lambda_{k}\right)=\left(\bigoplus_{s=0}^{k} \pi_{k}^{i+s, s}\left(H_{k+1}^{i+s}\right)\right) \oplus D_{k}^{i+k+1}\left(\lambda_{k}\right) / D_{k}^{i+k+2}\left(\lambda_{k}\right) \quad \text { if } i<0, \\
& D_{k}^{i}\left(\lambda_{k}\right)=\left(\bigoplus_{s=i}^{k-1} H_{k+1}^{i}\right) \oplus D_{k}^{k}\left(\lambda_{k}\right) \quad \text { if } \quad 0 \leq i<k .
\end{aligned}
$$


Let $\operatorname{pr}_{i}^{\mathcal{H}_{k+1}}$ be the projection of $D_{k}^{i}\left(\lambda_{k}\right) / D_{k}^{i+k+2}\left(\lambda_{k}\right)$ to $D_{k}^{i+k+1}\left(\lambda_{k}\right) / D_{k}^{i+k+2}\left(\lambda_{k}\right)$ corresponding to the splitting (4.9) if $i<0$ or the projection of $D_{k}^{i}\left(\lambda_{k}\right)$ to $H_{k+1}^{k-1}$ corresponding to the splitting (4.10) if $0 \leq i<k$. Given vectors $v_{1} \in \mathfrak{g}^{-1}$ and $v_{2} \in \mathfrak{g}^{i}$ take two vector fields $Y_{1}$ and $Y_{2}$ in a neighborhood $U_{k}$ of $\lambda_{k}$ in $P^{k}$ such that for any $\tilde{\lambda}_{k}=\left(\tilde{\lambda}_{k-1}, \tilde{\varphi}_{k}\right) \in U_{k}$, where $\tilde{\varphi}_{k} \in \bigoplus_{i<k} \operatorname{Hom}\left(\mathfrak{g}^{i}, D_{k-1}^{i}\left(\lambda_{k-1}\right) / D_{k-1}^{i+k+1}\left(\lambda_{k-1}\right)\right)$, one has

$$
\begin{aligned}
& \Pi_{k_{*}} Y_{1}\left(\tilde{\lambda}_{k}\right)=\tilde{\varphi}_{k}\left(v_{1}\right), \quad \Pi_{k_{*}} Y_{2}\left(\tilde{\lambda}_{k}\right) \equiv \tilde{\varphi}_{k}\left(v_{2}\right) \quad \bmod D_{k-1}^{i+k+1}\left(\lambda_{k-1}\right), \\
& Y_{1}(\lambda)=\varphi^{\mathcal{H}_{k+1}}\left(v_{1}\right), \quad Y_{2}(\lambda) \equiv \varphi^{\mathcal{H}_{k+1}}\left(v_{2}\right) \quad \bmod D_{k}^{i+k+2}(\lambda) .
\end{aligned}
$$

Then set

$$
C_{\mathcal{H}^{k+1}}^{k}\left(v_{1}, v_{2}\right) \stackrel{\text { def }}{=} \begin{cases}\bar{\omega}_{k}^{i+k}\left(\operatorname{pr}_{i-1}^{\mathcal{H}_{k+1}}\left(\left[Y_{1}, Y_{2}\right]\right)\right) & \text { if } i<0, \\ \omega_{k}^{k-1}\left(\operatorname{pr}_{i-1}^{\mathcal{H}_{k+1}}\left(\left[Y_{1}, Y_{2}\right]\right)\right) & \text { if } 0 \leq i<k .\end{cases}
$$

As in the case of the first prolongation, $C_{\mathcal{H}}^{k}\left(v_{1}, v_{2}\right)$ does not depend on the choice of vector fields $Y_{1}$ and $Y_{2}$, satisfying (4.11). Indeed, assume that $\widetilde{Y}_{1}$ and $\widetilde{Y}_{2}$ is another pair of vector fields in a neighborhood of $\lambda_{k}$ in $P^{k}$ such that $\widetilde{Y}_{1}$ is a section of $D_{k}^{-1}, \widetilde{Y}_{2}$ is a section of $D_{k}^{i}$, and they satisfy (4.11) with $Y_{1}, Y_{2}$ replaced by $\widetilde{Y}_{1}, \widetilde{Y}_{1}$. Then

$$
\tilde{Y}_{1}=Y_{1}+Z_{1}, \quad \tilde{Y}_{2}=Y_{2}+Z_{2},
$$

where $Z_{1}$ is a section of the distribution $D_{k}^{k}$ such that $Z_{1}\left(\lambda_{k}\right)=0$ and $Z_{2}$ is a section of the distribution $D_{k}^{\min \{i+k+1, k\}}$ such that $Z_{2}\left(\lambda_{k}\right) \in D_{k}^{\min \{i+k+1, k\}+1}\left(\lambda_{k}\right)$. Then $\left[Y_{1}, Z_{2}\right]\left(\lambda_{k}\right) \in$ $D_{k}^{\min \{i+k+1, k\}}\left(\lambda_{k}\right)$ and $\left[Y_{2}, Z_{1}\right](\lambda) \in D_{k}^{\min \{i+k+1, k\}}\left(\lambda_{k}\right)$. This together with the fact that $\left[Z_{1}, Z_{2}\right]$ is a section of $D_{k}^{\min \{i+k+1, k\}+1}$ implies that

$$
\left[\widetilde{Y}_{1}, \widetilde{Y}_{2}\right](\lambda) \equiv\left[Y_{1}, Y_{2}\right] \quad \bmod D_{k}^{\min \{i+k+1, k\}}(\lambda)
$$

From (4.12) it follows that the structure function is independent of the choice of vector fields $Y_{1}$ and $Y_{2}$.

Now take another tuple $\widetilde{\mathcal{H}}_{k+1}=\left\{\widetilde{H}_{k+1}^{i}\right\}_{i<k}$ such that

1. for $i<0$ the space $\widetilde{H}_{k+1}^{i}$ is a complement of $D_{k}^{i+k+1}\left(\lambda_{k}\right) / D_{k}^{i+k+2}\left(\lambda_{k}\right)$ in $\left(\Pi_{k}^{i} \circ \pi_{k}^{i}\right)^{-1}\left(H_{k}^{i}\right) \subset$ $D_{k}^{i}\left(\lambda_{k}\right) / D_{k}^{i+k+2}\left(\lambda_{k}\right)$,

$$
\left(\Pi_{k}^{i} \circ \pi_{k}^{i}\right)^{-1}\left(H_{k}^{i}\right)=D_{k}^{i+k+1}\left(\lambda_{k}\right) / D_{k}^{i+k+2}\left(\lambda_{k}\right) \oplus \widetilde{H}_{k+1}^{i}
$$

2. for $0 \leq i<k$ the space $\widetilde{H}_{k+1}^{i}$ is a complement of $D_{k}^{k}\left(\lambda_{k}\right)$ in $\left(\Pi_{k}^{i}\right)^{-1}\left(H_{k}^{i}\right)$,

$$
\left(\Pi_{k}^{i}\right)^{-1}\left(H_{k}^{i}\right)=D_{k}^{k}\left(\lambda_{k}\right) \oplus \widetilde{H}_{k+1}^{i}
$$

How are the structure functions $C_{\mathcal{H}_{k+1}}^{k}$ and $C_{\widetilde{\mathcal{H}}_{k+1}}^{k}$ related? By construction, for any vector $v \in \mathfrak{g}^{i}$ the vector $\varphi^{\widetilde{\mathcal{H}}_{k+1}}(v)-\varphi^{\mathcal{H}_{k+1}}(v)$ belongs to $D_{k}^{i+k+1}\left(\lambda_{k}\right) / D_{k}^{i+k+2}\left(\lambda_{k}\right)$, for $i<0$, and to $D_{k}^{k}\left(\lambda_{k}\right)$, for $0 \leq i<k$. Let

$$
f_{\mathcal{H}_{k+1} \widetilde{\mathcal{H}}_{k+1}}(v) \stackrel{\text { def }}{=} \begin{cases}\bar{\omega}_{k}^{i+k+1}\left(\varphi^{\widetilde{\mathcal{H}}_{k+1}}(v)-\varphi^{\mathcal{H}_{k+1}}(v)\right) & \text { if } v \in \mathfrak{g}^{i} \text { with } i<-1, \\ I_{\lambda}^{-1}\left(\varphi^{\widetilde{\mathcal{H}}_{k+1}}(v)-\varphi^{\mathcal{H}_{k+1}}(v)\right) & \text { if } v \in \mathfrak{g}^{i} \text { with }-1 \leq i<k .\end{cases}
$$


Then

$$
f_{\mathcal{H}_{k+1} \widetilde{\mathcal{H}}_{k+1}} \in \bigoplus_{i<0} \operatorname{Hom}\left(\mathfrak{g}^{i}, \mathfrak{g}^{i+k+1}\right) \oplus \bigoplus_{i=0}^{k-1} \operatorname{Hom}\left(\mathfrak{g}^{i}, \mathfrak{g}^{k}\right)
$$

In the opposite direction, it is clear that for any $f \in \bigoplus_{i<0} \operatorname{Hom}\left(\mathfrak{g}^{i}, \mathfrak{g}^{i+k+1}\right) \oplus \bigoplus_{i=0}^{k-1} \operatorname{Hom}\left(\mathfrak{g}^{i}, \mathfrak{g}^{k}\right)$, there exists a tuple $\widetilde{\mathcal{H}}_{k+1}=\left\{\widetilde{H}_{k+1}^{i}\right\}_{i<k}$ satisfying (4.13) and (4.14) and such that $f=f_{\mathcal{H}_{k+1}} \widetilde{\mathcal{H}}_{k+1}$. Further, let $\mathcal{A}_{k}$ be as in (4.8) and define a map

$$
\partial_{k}: \bigoplus_{i<0} \operatorname{Hom}\left(\mathfrak{g}^{i}, \mathfrak{g}^{i+k+1}\right) \oplus \bigoplus_{i=0}^{k-1} \operatorname{Hom}\left(\mathfrak{g}^{i}, \mathfrak{g}^{k}\right) \rightarrow \mathcal{A}_{k}
$$

by

$$
\begin{aligned}
\partial_{k} f( & \left.v_{1}, v_{2}\right) \\
\quad & = \begin{cases}{\left[f\left(v_{1}\right), v_{2}\right]+\left[v_{1}, f\left(v_{2}\right)\right]-f\left(\left[v_{1}, v_{2}\right]\right)} & \text { if } v_{1} \in \mathfrak{g}^{-1}, v_{2} \in \mathfrak{g}^{i}, i<0, \\
{\left[v_{1}, f\left(v_{2}\right)\right]} & \text { if } v_{1} \in \mathfrak{g}^{-1}, v_{2} \in \mathfrak{g}^{i}, 0 \leq i<k-1,\end{cases}
\end{aligned}
$$

where the brackets [,] are as in the algebraic universal prolongation $\mathfrak{g}\left(\mathfrak{m}, \mathfrak{g}^{0}\right)$. For $k=0$ this definition coincides with the definition of the generalized Spencer operator for the first prolongation given in the previous section.

The reason for introducing the operator $\partial_{k}$ is that the following generalization of identity (3.8) holds:

$$
C_{\widetilde{\mathcal{H}}_{k+1}}^{k}=C_{\mathcal{H}_{k+1}}^{k}+\partial_{k} f_{\mathcal{H}_{k+1} \tilde{\mathcal{H}}_{k+1}}
$$

A verification of this identity for pairs $\left(v_{1}, v_{2}\right)$, where $v_{1} \in \mathfrak{g}^{-1}$ and $v_{2} \in \mathfrak{g}^{i}$ with $i<0$, is completely analogous to the proof of Proposition 3.1. For $i \geq 0$ one has to use the inductive assumption that the restrictions $\left.\varphi_{l}\right|_{\mathfrak{q}^{i}}$ are the same for all $\lambda_{l}$ from the same fiber (see item 3 from the list of properties satisfied by $P^{l}$ in the beginning of this section) and the splitting (4.10).

Now we proceed as in Sections 2 and 3. Fix a subspace

$$
\mathcal{N}_{k} \subset \mathcal{A}_{k}
$$

which is complementary to $\operatorname{Im} \partial_{k}$,

$$
\mathcal{A}_{k}=\operatorname{Im} \partial_{k} \oplus \mathcal{N}_{k}
$$

As above, the subspace $\mathcal{N}_{k}$ defines the normalization conditions for the first prolongation. Then from the splitting (4.16) it follows trivially that there exists a tuple $\mathcal{H}_{k+1}=\left\{H_{k+1}^{i}\right\}_{i<k}$, satisfying (4.6) and (4.7), such that

$$
C_{\mathcal{H}_{k+1}}^{k} \in \mathcal{N}_{k}
$$

and $C_{\widetilde{\mathcal{H}}_{k+1}}^{k} \in \mathcal{N}_{k}$ for a tuple $\widetilde{H}_{k+1}=\left\{H_{k+1}^{i}\right\}_{i<k}$, satisfying (4.13) and (4.14), if and only if $f_{\mathcal{H}_{k+1} \tilde{\mathcal{H}}_{k+1}} \in$ ker $\partial_{k}$. Note also that

$$
f \in \text { ker }\left.\partial_{k} \Rightarrow f\right|_{\mathfrak{g}^{i}}=0 \quad \forall 0 \leq i \leq k-1 .
$$


In other words,

$$
\text { ker } \partial_{k} \subset \bigoplus_{i<0} \operatorname{Hom}\left(\mathfrak{g}^{i}, \mathfrak{g}^{i+k+1}\right) \text {. }
$$

Indeed, if $f \in$ ker $\partial_{k}$, then by (4.15) for any $v_{1} \in \mathfrak{g}^{-1}$ and $v_{2} \in \mathfrak{g}^{i}$ with $0 \leq i \leq k-1$ one has

$$
\left[v_{1}, f\left(v_{2}\right)\right]=-f\left(v_{2}\right) v_{1}=0
$$

In other words, $\left.f\left(v_{2}\right)\right|_{\mathfrak{g}^{-1}}=0$ (recall that $\left.f\left(v_{2}\right) \in \mathfrak{g}^{k} \subset \bigoplus_{i<0} \operatorname{Hom}\left(\mathfrak{g}^{i}, \mathfrak{g}^{i+k}\right)\right)$. Since $\mathfrak{g}^{-1}$ generates the whole symbol $\mathfrak{m}$ we see that $f\left(v_{2}\right)=0$ holds for any $v_{2} \in \mathfrak{g}^{i}$ with $0 \leq i \leq k-1$. This proves that (4.17).

Further, comparing (4.15) and (4.18) with (4.2) and using again the fact that $\mathfrak{g}^{-1}$ generates the whole symbol $\mathfrak{m}$ we obtain

$$
\text { ker } \partial_{k}=\mathfrak{g}^{k+1} \text {. }
$$

The $(k+1)$-st (geometric) prolongation of the bundle $P^{0}$ is the bundle $P^{k+1}$ over $P^{k}$ defined by

$$
P^{k+1}=\left\{\left(\lambda_{k}, \mathcal{H}_{k+1}\right): \lambda_{k} \in P^{k}, C_{\mathcal{H}_{k+1}}^{k} \in \mathcal{N}_{k}\right\}
$$

Equivalently,

$$
P^{k+1}=\left\{\left(\lambda, \varphi^{\mathcal{H}_{k+1}}\right): \lambda_{k} \in P^{k}, C_{\mathcal{H}_{k+1}}^{k} \in \mathcal{N}_{k}\right\} .
$$

It is a principal bundle with the abelian structure group $G^{k+1}$ of all maps $A \in \bigoplus_{i \leq k} \operatorname{Hom}\left(\mathfrak{g}^{i}\right.$, $\left.\mathfrak{g}^{i} \oplus \mathfrak{g}^{i+k+1}\right)$ such that

$$
\left.A\right|_{\mathfrak{g}^{i}}= \begin{cases}\operatorname{Id}_{\mathfrak{g}^{i}}+T_{i} & \text { if } i<0, \\ \operatorname{Id}_{\mathfrak{g}^{i}} & \text { if } 0 \leq i \leq k,\end{cases}
$$

where $T_{i} \in \operatorname{Hom}\left(\mathfrak{g}^{i}, \mathfrak{g}^{i+k+1}\right)$ and $\left(T_{-\mu}, \ldots, T_{-1}\right) \in \mathfrak{g}^{k+1}$. The right action $R_{A}^{k+1}$ of $A \in G^{k+1}$ on a fiber of $P^{k+1}$ is defined by $R_{A}^{k+1}\left(\varphi^{\mathcal{H}_{k+1}}\right)=\varphi^{\mathcal{H}_{k+1}} \circ A$. Obviously, $G^{k+1}$ is an abelian group of dimension equal to $\operatorname{dim} \mathfrak{g}^{k+1}$. It is easy to see that the bundle $P^{k+1}$ is constructed so that the Properties 1-4, formulated in the beginning of the present section, hold for $l=k+1$ as well.

Finally, assume that there exists $\bar{l} \geq 0$ such that $\mathfrak{g}^{\bar{l}} \neq 0$ but $\mathfrak{g}^{\bar{l}+1}=0$. Since the symbol $\mathfrak{m}$ is fundamental, it follows that $\mathfrak{g}^{l}=0$ for all $l>\bar{l}$. Hence, for all $l>\bar{l}$ the fiber of $P^{l}$ over a point $\lambda_{l-1} \in P^{l-1}$ is a single point belonging to $\bigoplus_{i=-\mu}^{l-1} \operatorname{Hom}\left(\mathfrak{g}^{i}, D_{l-1}^{i}\left(\lambda_{l-1}\right) / D_{l-1}^{i+l+1}\left(\lambda_{l-1}\right)\right)$, where, as before, $\mu$ is the degree of nonholonomy of the distribution $D$. Moreover, by our assumption, $D_{l}^{i}=0$ if $l \geq \bar{l}$ and $i \geq \bar{l}$. Therefore, if $l=\bar{l}+\mu$, then $i+l+1>\bar{l}$ for $i \geq-\mu$ and the fiber of $P^{l}$ over $P^{l}$ is an element of $\operatorname{Hom}\left(\bigoplus_{i=-\mu}^{l-1} \mathfrak{g}^{i}, T_{\lambda_{l-1}} P^{l-1}\right)$. In other words, $P^{\bar{l}+\mu}$ defines a canonical frame on $P^{\bar{l}+\mu-1}$. But all bundles $P^{l}$ with $l \geq \bar{l}$ are identified one with each other by the canonical projections (which are diffeomorphisms in that case). As a conclusion we get an alternative proof of the main result of the Tanaka paper [10]:

Theorem 4.1. If the $(\bar{l}+1)$-st algebraic prolongation of the graded Lie algebra $\mathfrak{m} \oplus \mathfrak{g}^{0}$ is equal to zero then for any structure $P^{0}$ of constant type $\left(\mathfrak{m}, \mathfrak{g}^{0}\right)$ there exists a canonical frame on the $\bar{l}$-th geometric prolongation $P^{\bar{l}}$ of $P^{0}$. 
The power of Theorem 4.1 is that it reduces the question of existence of a canonical frame for a structure of constant type $\left(\mathfrak{m}, \mathfrak{g}^{0}\right)$ to the calculation of the universal algebraic prolongation of the algebra $\mathfrak{m} \oplus \mathfrak{g}^{0}$. But the latter is pure Linear Algebra: each consecutive algebraic prolongation is determined by solving the system of linear equations given by (4.2). Let us demonstrate this algebraic prolongation procedure in the case of the equivalence of second order ordinary differential equations with respect to the group of point transformations (see Example 5 in Subsection 1.3). The result of this prolongation is very well known using the structure theory of simple Lie algebras (see discussions below), but this is one of the few nontrivial examples, where explicit calculations of algebraic prolongation can be written down in detail within one and a half pages.

Continuation of Example 5. Recall that our geometric structure here is a contact distribution $D$ on a 3-dimensional manifold endowed with two distinguished transversal line subdistributions. The symbol of $D$ is isomorphic to the 3 -dimensional Heisenberg algebra $\eta_{3}$ with grading $\mathfrak{g}^{-1} \oplus \mathfrak{g}^{-2}$, where $\mathfrak{g}^{-2}$ is the center of $\eta_{3}$. Besides, the plane $\mathfrak{g}^{-1}$ is endowed with two distinguished transversal lines $\ell_{1}$ and $\ell_{2}$. Let $X_{1}$ and $X_{2}$ be vectors spanning $\ell_{1}$ and $\ell_{2}$, respectively, and let $X_{3}=\left[X_{1}, X_{2}\right]$. Let $g^{0}$ be the algebra of all derivations on $\eta_{3}$ preserving the grading and the lines $\ell_{1}$ and $\ell_{2}$. Then

$$
\mathfrak{g}^{0}=\operatorname{span}\left\{\Lambda_{1}^{0}, \Lambda_{2}^{0}\right\}
$$

where

$$
\Lambda_{1}^{0}\left(X_{1}\right)=X_{1}, \quad \Lambda_{1}^{0}\left(X_{2}\right)=X_{2}, \quad \Lambda_{2}^{0}\left(X_{1}\right)=X_{1}, \quad \Lambda_{2}^{0}\left(X_{2}\right)=-X_{2} .
$$

Using the fact that $\Lambda_{i}^{0}$ is a derivation, we also have

$$
\Lambda_{1}^{0}\left(X_{3}\right)=2 X_{3}, \quad \Lambda_{2}^{0}\left(X_{3}\right)=0 .
$$

a) Calculation of $\mathfrak{g}^{1}$. Given $\delta^{1} \in \operatorname{Hom}\left(\mathfrak{g}^{-1}, \mathfrak{g}^{0}\right) \oplus \operatorname{Hom}\left(\mathfrak{g}^{-2}, \mathfrak{g}^{-1}\right)$ we have

$$
\delta^{1}\left(X_{1}\right)=\alpha_{11} \Lambda_{1}^{0}+\alpha_{12} \Lambda_{2}^{0}, \quad \delta^{1}\left(X_{2}\right)=\alpha_{21} \Lambda_{1}^{0}+\alpha_{22} \Lambda_{2}^{0}
$$

for some $\alpha_{i j}, 1 \leq i, j \leq 2$. If $\delta^{1} \in \mathfrak{g}^{1}$, then (4.19) yields

$$
\begin{aligned}
\delta^{1}\left(X_{3}\right) & =\left[\delta^{1}\left(X_{1}\right), X_{2}\right]+\left[X_{1}, \delta^{1}\left(X_{2}\right)\right] \\
& =\left(\alpha_{11} \Lambda_{1}^{0}+\alpha_{12} \Lambda_{2}^{0}\right)\left(X_{2}\right)-\left(\alpha_{21} \Lambda_{1}^{0}+\alpha_{22} \Lambda_{2}^{0}\right)\left(X_{1}\right)=-\left(\alpha_{21}+\alpha_{22}\right) X_{1}+\left(\alpha_{11}-\alpha_{12}\right) X_{2} .
\end{aligned}
$$

Using (4.20) and (4.22), we have

$$
\begin{aligned}
0 & =\delta^{1}\left(\left[X_{1}, X_{3}\right]\right)=\left[\delta^{1}\left(X_{1}\right), X_{3}\right]+\left[X_{1}, \delta^{1}\left(X_{3}\right)\right] \\
& =\left(\alpha_{11} \Lambda_{1}^{0}+\alpha_{12} \Lambda_{2}^{0}\right)\left(X_{3}\right)+\left(\alpha_{11}-\alpha_{12}\right) X_{3}=\left(3 \alpha_{11}-\alpha_{12}\right) X_{3},
\end{aligned}
$$

which implies that $\alpha_{12}=3 \alpha_{11}$. In the same way, from the identities $0=\delta^{1}\left[X_{2}, X_{3}\right]=$ $\left[\delta^{1}\left(X_{2}\right), X_{3}\right]+\left[X_{2}, \delta^{1}\left(X_{3}\right)\right]$ one obtains easily that $\alpha_{22}=-3 \alpha_{21}$. This completes the verification of conditions for $\delta^{1}$ to be in $\mathfrak{g}^{1}$. Hence

$$
\mathfrak{g}^{1}=\operatorname{span}\left\{\Lambda_{1}^{1}, \Lambda_{2}^{1}\right\},
$$

where $\Lambda_{1}^{1}, \Lambda_{2}^{1} \in \operatorname{Hom}\left(\mathfrak{g}^{-1}, \mathfrak{g}^{0}\right) \oplus \operatorname{Hom}\left(\mathfrak{g}^{-2}, \mathfrak{g}^{-1}\right)$ such that

$$
\begin{array}{lll}
\Lambda_{1}^{1}\left(X_{1}\right)=\Lambda_{1}^{0}+3 \Lambda_{2}^{0}, & \Lambda_{1}^{1}\left(X_{2}\right)=0, & \Lambda_{1}^{1}\left(X_{3}\right)=-2 X_{2}, \\
\Lambda_{2}^{1}\left(X_{1}\right)=0, & \Lambda_{2}^{1}\left(X_{2}\right)=\Lambda_{1}^{0}-3 \Lambda_{2}^{0}, & \Lambda_{2}^{1}\left(X_{3}\right)=2 X_{1} .
\end{array}
$$


$\left(\Lambda_{1}^{1}\right.$ corresponds to $\delta^{1}$ as in (4.21) and (4.22) with $\alpha_{11}=1$ and $\alpha_{21}=0$, while $\Lambda_{2}^{1}$ corresponds to $\delta^{1}$ with $\alpha_{11}=0$ and $\alpha_{21}=1$ in the same formulas).

b) Calculation of $\mathfrak{g}^{2}$. Given $\delta^{2} \in \operatorname{Hom}\left(\mathfrak{g}^{-1}, \mathfrak{g}^{1}\right) \oplus \operatorname{Hom}\left(\mathfrak{g}^{-2}, \mathfrak{g}^{0}\right)$ we have

$$
\delta^{2}\left(X_{1}\right)=\beta_{11} \Lambda_{1}^{1}+\beta_{12} \Lambda_{2}^{1}, \quad \delta^{2}\left(X_{2}\right)=\beta_{21} \Lambda_{1}^{1}+\beta_{22} \Lambda_{2}^{1}
$$

for some $\beta_{i j}, 1 \leq i, j \leq 2$. If $\delta^{2} \in \mathfrak{g}^{2}$, then (4.23) implies

$$
\begin{aligned}
\delta^{2}\left(X_{3}\right) & =\left[\delta^{2}\left(X_{1}\right), X_{2}\right]+\left[X_{1}, \delta^{2}\left(X_{2}\right)\right] \\
& =\left(\beta_{11} \Lambda_{1}^{1}+\beta_{12} \Lambda_{2}^{1}\right)\left(X_{2}\right)-\left(\beta_{21} \Lambda_{1}^{1}+\beta_{22} \Lambda_{2}^{1}\right)\left(X_{1}\right)=\left(\beta_{12}-\beta_{21}\right) \Lambda_{1}^{0}-3\left(\beta_{12}+\beta_{21}\right) \Lambda_{2}^{0} .
\end{aligned}
$$

Using (4.23) and (4.24), we have

$$
\begin{aligned}
0 & =\delta^{2}\left(\left[X_{1}, X_{3}\right]\right)=\left[\delta^{2}\left(X_{1}\right), X_{3}\right]+\left[X_{1}, \delta^{2}\left(X_{3}\right)\right] \\
& =\left(\beta_{11} \Lambda_{1}^{1}+\beta_{12} \Lambda_{2}^{1}\right)\left(X_{3}\right)-\left(\beta_{12}-\beta_{21}\right) \Lambda_{1}^{0}\left(X_{1}\right)+3\left(\beta_{12}+\beta_{21}\right) \Lambda_{2}^{0}\left(X_{1}\right) \\
& =4\left(\beta_{12}+\beta_{21}\right) X_{1}-2 \beta_{11} X_{2},
\end{aligned}
$$

which implies that

$$
\beta_{11}=0, \quad \beta_{21}=-\beta_{12} .
$$

Similarly, the identities $0=\delta^{2}\left[X_{2}, X_{3}\right]=\left[\delta^{2}\left(X_{2}\right), X_{3}\right]+\left[X_{2}, \delta^{2}\left(X_{3}\right)\right]$ implies $\beta_{22}=0$ in addition to $(4.25)$. This completes the verifications of conditions for $\delta^{2}$ to be in $\mathfrak{g}^{2}$. Hence

$$
\mathfrak{g}^{2}=\operatorname{span}\{\Lambda\},
$$

where $\Lambda \in \operatorname{Hom}\left(\mathfrak{g}^{-1}, \mathfrak{g}^{1}\right) \oplus \operatorname{Hom}\left(\mathfrak{g}^{-2}, \mathfrak{g}^{0}\right)$ is defined by

$$
\Lambda\left(X_{1}\right)=\Lambda_{2}^{1}, \quad \Lambda\left(X_{2}\right)=-\Lambda_{1}^{1}, \quad \Lambda\left(X_{3}\right)=2 \Lambda_{1}^{0} .
$$

c) Calculation of $\mathfrak{g}^{3}$. Given $\delta^{3} \in \operatorname{Hom}\left(\mathfrak{g}^{-1}, \mathfrak{g}^{2}\right) \oplus \operatorname{Hom}\left(\mathfrak{g}^{-2}, \mathfrak{g}^{1}\right)$ such that

$$
\delta^{3}\left(X_{1}\right)=\gamma_{1} \Lambda, \quad \delta^{2}\left(X_{2}\right)=\gamma_{2} \Lambda .
$$

for some $\gamma_{i}, i=1,2$. If $\delta^{3} \in \mathfrak{g}^{2}$, then (4.26) implies $\delta^{3}\left(X_{1}\right)=-\gamma_{1} \Lambda_{1}^{1}-\gamma_{2} \Lambda_{2}^{1}$. From the identities $0=\delta^{3}\left[X_{1}, X_{3}\right]=\left[\delta^{3}\left(X_{1}\right), X_{3}\right]+\left[X_{1}, \delta^{3}\left(X_{3}\right)\right]$ it follows easily that $\gamma_{1}=0$. From the identities $0=\delta^{3}\left[X_{2}, X_{3}\right]=\left[\delta^{3}\left(X_{2}\right), X_{3}\right]+\left[X_{2}, \delta^{3}\left(X_{3}\right)\right]$ it follows easily that $\gamma_{2}=0$. Hence,

$$
\mathfrak{g}^{3}=0 \text {. }
$$

Thus the algebraic universal prolongation $\mathfrak{g}\left(\eta_{3}, \mathfrak{g}^{0}\right)=\mathfrak{g}^{-2} \oplus \mathfrak{g}^{-1} \oplus \mathfrak{g}^{0} \oplus \mathfrak{g}^{1} \oplus \mathfrak{g}^{2}$ is 8-dimensional. Therefore, fixing the normalization conditions at each step, one can construct the first and the second geometric prolongations $P^{1}$ and $P^{2}$, and for any contact distribution $D$ on 3 -dimensional manifold endowed with two distinguished transversal line sub-distributions there is a canonical frame on the 8-dimensional bundle $P^{2}$.

Let us look at the algebra $\mathfrak{g}\left(\eta_{3}, \mathfrak{g}^{0}\right)$ in more detail. Applying (4.3) inductively, we see that all nonzero brackets of elements $\Lambda_{1}^{0}, \Lambda_{2}^{0}, \Lambda_{1}^{1}, \Lambda_{2}^{1}, \Lambda$ (spanning the subalgebra of elements with nonzero weights of $\left.\mathfrak{g}\left(\eta_{3}, \mathfrak{g}^{0}\right)\right)$ are as follows:

$$
\left[\Lambda_{1}^{1}, \Lambda_{1}^{0}\right]=\Lambda_{1}^{1}, \quad\left[\Lambda_{1}^{1}, \Lambda_{2}^{0}\right]=\Lambda_{1}^{1}, \quad\left[\Lambda_{2}^{1}, \Lambda_{1}^{0}\right]=\Lambda_{1}^{1}, \quad\left[\Lambda_{2}^{1}, \Lambda_{1}^{0}\right]=-\Lambda_{2}^{1}, \quad\left[\Lambda_{1}^{1}, \Lambda_{2}^{1}\right]=2 \Lambda .
$$

Considering all products in $\mathfrak{g}\left(\eta_{3}, \mathfrak{g}^{0}\right)$ it is not hard to see that $\mathfrak{g}\left(\eta_{3}, \mathfrak{g}^{0}\right)$ is isomorphic to $\mathfrak{s} \mathfrak{l}(3, \mathbb{R})$. Indeed, if we denote by $E_{i j}$ the $3 \times 3$-matrix such that its $(i, j)$ entry is equal to 1 and all other entries vanish, then the following mapping is an isomorphism of algebras $\mathfrak{g}\left(\eta_{3}, \mathfrak{g}^{0}\right)$ and $\mathfrak{s l}(3, \mathbb{R})$ :

$$
\begin{aligned}
& X_{1} \mapsto E_{12}, \quad X_{2} \mapsto E_{23}, \quad X_{3} \mapsto E_{13}, \\
& \Lambda_{1}^{1} \mapsto-2 E_{21}, \quad \Lambda_{2}^{1} \mapsto-2 E_{23}, \quad \Lambda \mapsto-2 E_{23}, \\
& \Lambda_{1}^{0}+3 \Lambda_{2}^{0} \mapsto 2\left(E_{11}-E_{22}\right), \quad \Lambda_{1}^{0}-3 \Lambda_{2}^{0} \mapsto 2\left(E_{22}-E_{33}\right) .
\end{aligned}
$$


As a matter of fact, here we are in the situation, when $\mathfrak{m} \oplus \mathfrak{g}^{0}$ is a subalgebra of elements of nonnegative weights (a parabolic subalgbebra) of a graded simple Lie algebra (in the considered case $\eta_{3} \oplus \mathfrak{g}^{0}$ is a Borel subalgebra of $\left.\mathfrak{s t}(3, \mathbb{R})\right)$. It was shown in [14] that, except for a few cases, the algebraic universal prolongation of a parabolic subalgebra of a simple Lie algebra is isomorphic to this simple Lie algebra.

This result can be applied also to the algebra $\mathfrak{m}_{(2,5)} \oplus \mathfrak{g}^{0}\left(\mathfrak{m}_{(2,5)}\right)$, corresponding to maximally nonholonomic rank 2 distributions in $\mathbb{R}^{5}$ (see Example 2 above). In this case $\mathfrak{m}_{(2,5)} \oplus \mathfrak{g}^{0}\left(\mathfrak{m}_{(2,5)}\right)$ is the subalgebra of elements of non-negative degree in the exceptional Lie algebra $G_{2}$, graded according to the coefficient of the short simple root. So, according to [14], the algebraic universal prolongation of $\mathfrak{m}_{(2,5)} \oplus \mathfrak{g}^{0}\left(\mathfrak{m}_{(2,5)}\right)$ is isomorphic to $G_{2}=\bigoplus_{i=-3}^{3} \mathfrak{g}^{i}$. This together with Theorem 4.1 implies that to any maximally nonholonomic rank 2 distribution in $\mathbb{R}^{5}$ one can assign a canonical frame on the bundle $P^{3}$ of dimension equal to $\operatorname{dim} G_{2}=14$. Note that this statement is still weaker than what Cartan proved in [1]. Indeed, Cartan provides explicit expressions for the coframe and finds the complete system of invariants, while Theorem 4.1 is only the existence statement.

Finally note that the construction of the bundles $P^{k}$ (and therefore of the canonical frame) depends on the choice of the normalization conditions given by spaces $\mathcal{N}_{k}$, as in (4.16). Under additional assumptions on the algebra $\mathfrak{g}\left(\mathfrak{m}, \mathfrak{g}^{0}\right.$ ) (for example, semisimplicity or existence of a special bilinear form) the spaces $\mathcal{N}_{k}$ themselves can be taken in a canonical way at each step of the prolongation procedure. This allows to construct canonical frames satisfying additional nice properties.

In particular, in another fundamental paper of Tanaka [11], it was shown that if the algebraic universal prolongation $\mathfrak{g}\left(\mathfrak{m}, \mathfrak{g}^{0}\right)$ is a semisimple Lie algebra, then the so-called $\mathfrak{g}\left(\mathfrak{m}, \mathfrak{g}^{0}\right)$-valued normal Cartan connection can be associated with a structure of type $\left(\mathfrak{m}, \mathfrak{g}^{0}\right)$. Roughly speaking, a Cartan connection gives the canonical frame which is compatible in a natural way with the whole algebra $\mathfrak{g}\left(\mathfrak{m}, \mathfrak{g}^{0}\right)$. This is a generalization of Cartan's results [1] on maximally nonholonomic rank 2 distributions in $\mathbb{R}^{5}$. Further, T. Morimoto [4] gave a general criterion (in terms of the algebra $\mathfrak{g}\left(\mathfrak{m}, \mathfrak{g}^{0}\right)$ ) for the existence of the normal Cartan connection for structures of type $\left(\mathfrak{m}, \mathfrak{g}^{0}\right)$.

All these developments are far beyond of the goals of the present note, so we do not want to address them in more detail here, referring the reader to the original papers.

\section{Acknowledgements}

I would like to thank my colleagues Joseph Landsberg, Colleen Robles, and Dennis The for encouraging me to give these lectures, and Boris Doubrov for stimulating discussions. I am also very grateful to the anonymous referees for numerous useful suggestions and corrections.

\section{References}

[1] Cartan É., Les systémes de Pfaff á cinq variables et les équations aux dérivées partielles du second ordre, Ann. Sci. École Norm. Sup. (3) 27 (1910), 109-192 (reprinted in his Oeuvres completes, Partie II, Vol. 2, Paris, Gautier-Villars, 1953, 927-1010).

[2] Cartan É., Sur les variétés à connexion projective, Bull. Soc. Math. France 54 (1924), 205-241.

[3] Kuzmich O., Graded nilpotent Lie algebras in low dimensions, Lobachevskii J. Math. 3 (1999), $147-184$.

[4] Morimoto T., Geometric structures on filtered manifolds, Hokkaido Math. J. 22 (1993), $263-347$.

[5] Morimoto T., Lie algebras, geometric structures, and differential equations on filtered manifolds, in Lie Groups, Geometric Structures and Differential Equations - One Hundred Years after Sophus Lie (Kyoto/Nara, 1999), Adv. Stud. Pure Math., Vol. 37, Math. Soc. Japan, Tokyo, 2002, 205-252. 
[6] Morimoto T., Cartan connection associated with a subriemannian structure, Differential Geom. Appl. 26 (2008), 75-78.

[7] Singer I.M., Sternberg S., The infinite groups of Lie and Cartan. I. The transitive groups, J. Analyse Math. 15 (1965), 1-114.

[8] Spencer D.C., Overdetermined systems of linear partial differential equations, Bull. Amer. Math. Soc. 75 (1969), 179-239.

[9] Sternberg S., Lectures on differential geometry, Prentice-Hall, Inc., Englewood Cliffs, N.J., 1964.

[10] Tanaka N., On differential systems, graded Lie algebras and pseudogroups, J. Math. Kyoto. Univ. 10 (1970), $1-82$.

[11] Tanaka N., On the equivalence problems associated with simple graded Lie algebras, Hokkaido Math. J. 8 (1979), 23-84.

[12] Tresse A., Détermination des invariants ponctuels de l'équation différentielle ordinaire du second ordre $y^{\prime \prime}=w\left(x, y, y^{\prime}\right)$, S. Hirkei, Leipzig, 1896.

[13] Tresse A., Sur les invariants différentielles des groupes continus de transformations, Acta Math. 18 (1894), $1-88$.

[14] Yamaguchi K., Differential systems associated with simple graded Lie algebras, in Progress in Differential Geometry, Adv. Stud. Pure Math., Vol. 22, Math. Soc. Japan, Tokyo, 1993, 413-494. 